\section{(C) OPEN ACCESS}

\title{
Guidelines for the investigation of chronic diarrhoea in adults: British Society of Gastroenterology, 3rd edition
}

\author{
Ramesh P Arasaradnam, ${ }^{1,2,3}$ Steven Brown, ${ }^{4}$ Alastair Forbes, ${ }_{1}^{5}$ Mark R Fox ${ }_{1}^{6,7}$ \\ Pali Hungin, ${ }^{8}$ Lawrence Kelman, ${ }^{9}$ Giles Major, ${ }^{10}$ Michelle $0^{\prime}$ Connor, ${ }^{9}$ Dave S Sanders, ${ }^{4}$ \\ Rakesh Sinha, ${ }^{11}$ Stephen Charles Smith, ${ }^{12}$ Paul Thomas, ${ }^{13}$ Julian R F Walters ${ }^{14}$
}

- Additional material is published online only. To view please visit the journal online (http://dx.doi.org/10.1136/ gutjnl-2017-315909).

For numbered affiliations see end of article.

\section{Correspondence to} Professor Ramesh P Arasaradnam, Department of Gastroenterology, University Hospitals Coventry \& Warwickshire, Coventry, CV2 2DX, UK; r.arasaradnam@ warwick.ac.uk

Received 21 December 2017 Revised 28 February 2018 Accepted 11 March 2018 Published Online First 13 April 2018
Check for updates

To cite: Arasaradnam RP Brown S, Forbes A, et al. Gut 2018:67:1380-1399.

\begin{abstract}
Chronic diarrhoea is a common problem, hence clear guidance on investigations is required. This is an updated guideline from 2003 for the investigations of chronic diarrhoea commissioned by the Clinical Services and Standards Committee of the British Society of Gastroenterology (BSG). This document has undergone significant revision in content through input by 13 members of the Guideline Development Group (GDG) representing various institutions. The GRADE system was used to appraise the quality of evidence and grading of recommendations.
\end{abstract}

These guidelines deal with clinical assessment in primary and secondary care of a patient with diarrhoea, the exclusion of cancer or inflammation, and detecting common disorders such as bile acid diarrhoea, microscopic colitis, lactose malabsorption or post radiation diarrhoea, together with rarer causes of malabsorption and surgical disorders as outlined in figure 1. Options for therapy are not dealt with as it is beyond the remit of this guideline, nor has the cost effectiveness of investigations been addressed due to paucity of available evidence.

\section{PREFACE}

\subsection{Purpose of guidelines}

The guidelines are directed at consultant gastroenterologists, gastrointestinal surgeons, specialist registrars in training and general practitioners, and refer specifically to adult not paediatric gastroenterology. Their purpose is to provide guidance on the best available methods of investigating symptoms of chronic diarrhoea. Given this broad symptom-based focus, the guidelines cover a wide range of gastroenterological conditions and are not intended as a comprehensive review of all aspects of the clinical conditions mentioned herein, but rather an attempt to rationalise the approach to investigation in the context of this common clinical scenario.

\subsection{Development of guidelines and rigour}

The guidelines were prepared following a comprehensive literature search by the guideline development group (GDG). The GDG membership comprises elected members of the BSG, Association of Coloproctology of Great Britain and Ireland, Association of Biochemistry UK, British Institute of Radiology, Primary Care Society for Gastroenterology, and nurse and patient representatives. Each main section was authored by a designated member of the GDG following a comprehensive review of the literature including NICE, American and European guidelines. This involved a review of electronic databases (Medline and PubMed) using keywords (in both British and American spelling) such as $((($ ('diarrhoea-predominant irritable bowel syndrome' OR 'chronic diarrhoea' OR 'functional diarrhoea' OR 'loose stools' OR 'faecal urgency' OR 'faecal incontinence' OR 'stool frequency') AND ((diarrhoea[Title/Abstract]) AND (investigation OR investigate OR diagnostic OR diagnosis) AND "2000'(Publication Date): ‘3000'(Publication Date)) AND English(Language)) NOT case reports (Publication Type))). Additional terms related to the specific conditions mentioned in the text (eg, coeliac disease, bile acid diarrhoea or malabsorption and small bowel bacterial overgrowth). The time frame for literature review was from 2002 to April 2017. A total of 1292 key papers and relevant abstracts in English in peer-reviewed journals were identified and read, and relevant work has been cited and referenced. An initial draft document was produced and subsequently reviewed and modified in accordance with the AGREE tool. ${ }^{1}$ Two face-to-face meetings as well as a further two telephone meetings were held in preparing this guideline. We used a modified Delphi system to a maximum of three rounds for any contentious issues. A final decision was made once agreement was reached by $70 \%$ of the group. The lead author acted as facilitator. The review was undertaken through submission to members of the BSG Clinical Standards Service Committee as well as to BSG Council and finally international peer review.

\subsection{Grading of recommendations}

In accordance with the BSG advice on production of guidelines, the GDG applied the GRADE system. ${ }^{2}$ Strength of recommendation was strong, moderate or weak. Where the recommendation was unanimous a 'strong' recommendation was used, and where the decision was by majority and the recommendation was moderate or weak, 'we suggest' was used. We graded the evidence based on levels 1-5 as per the Oxford Centre for Evidence Based Medicine. ${ }^{3}$ In brief, Level $1 \mathrm{a}-\mathrm{c}$ ranges from systematic reviews with homogeneity, individual 


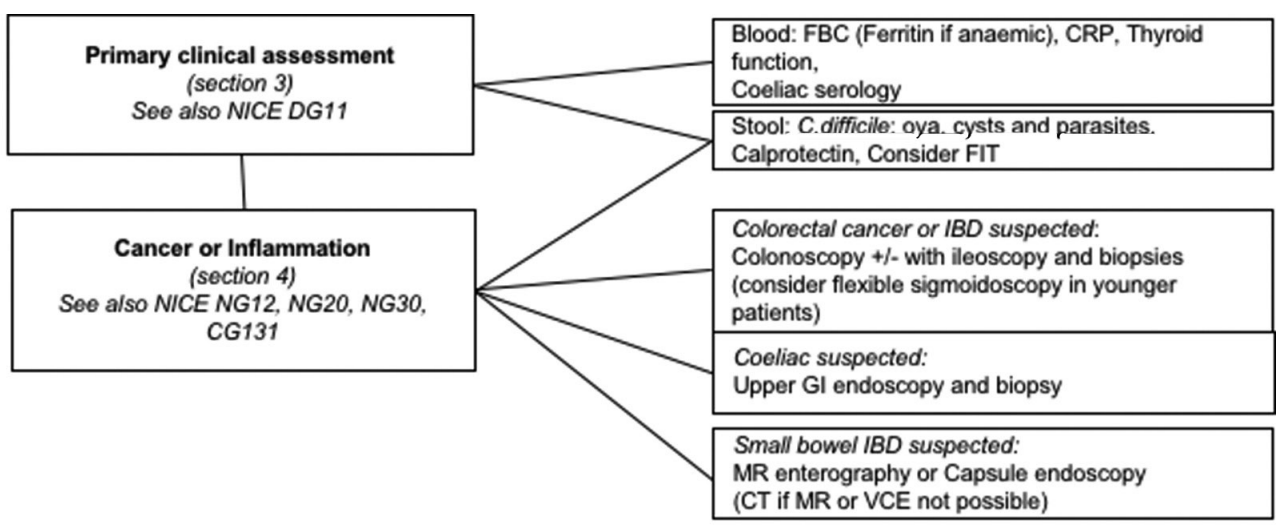

Consider

1) Positive diagnosis of diarrhoea-predominant irritable bowel syndrome (IBS-D) or functional diarrhoea

2) Specialist review -- especially for severe, persistent or atypical symptoms

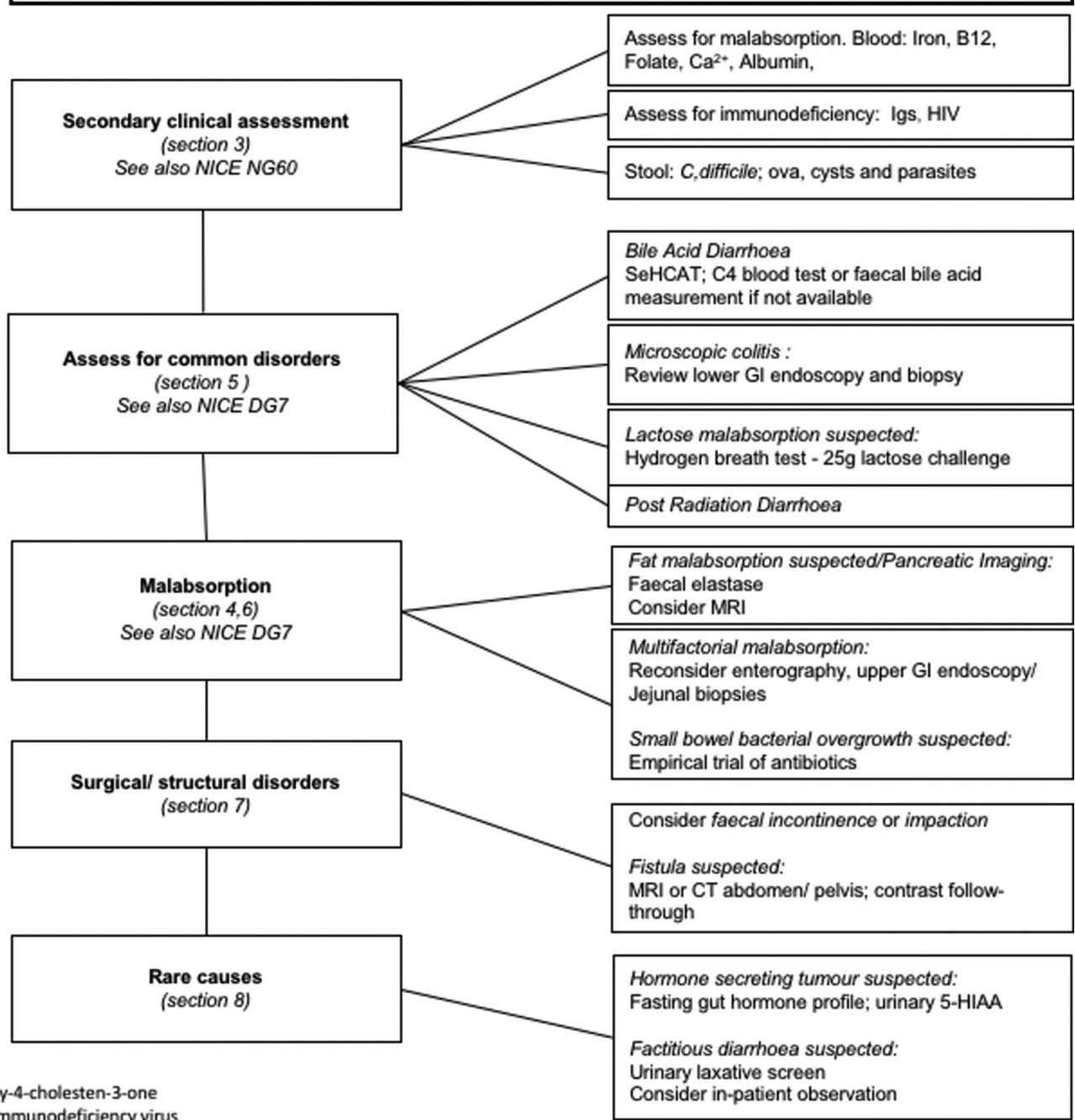

C4 - 7a-hydroxy-4-cholester

HIV - Human Immunodeficiency virus

Igs - Immunoglobulins

FIT - Faecal Immunochemical test for haemoglobin

SeHCAT - selenium 75 homotaurocholate

VCE - Video capsule endoscopy

5-HIAA - Hydroxyindole acetic acid

Figure 1 Algorithm for the investigation of chronic diarrhoea based on clinical differential diagnosis.

randomised controlled trials (RCTs); Level $2 \mathrm{a}-\mathrm{c}$ ranges from systematic reviews of cohort studies, low quality RCTs and outcomes research; Level $3 \mathrm{a}-\mathrm{b}$ ranges from systematic reviews with heterogeneity and individual case control studies; Level
4 are poor quality cohort or case series; and Level 5 is expert opinion without critical appraisal.

Specific RCTs addressing the investigation of "chronic diarrhoea' are absent. Thus our grading of evidence was based on 
We recommend the following investigation algorithm based on the clinical differential diagnosis outlined in figure 1.

\section{Clinical assessment}

- We recommend a careful detailed history to plan investigations (Grade of evidence level 1, Strength of recommendation strong).

- We recommend screening blood tests for the exclusion of anaemia, coeliac disease, etc as well as stool tests for inflammation (Grade of evidence level 1, Strength of recommendation strong).

- We recommend making a positive diagnosis of irritable bowel syndrome (IBS) following basic blood and stool screening tests (Grade of evidence level 2, Strength of recommendation strong).

\section{Cancer or inflammation}

- We recommend excluding colorectal cancer in those with altered bowel habittrectal bleeding by colonoscopy (Grade of evidence level 1 , Strength of recommendation strong).

- We suggest use of testing for faecal blood loss by faecal immunochemical testing in primary or secondary care, either as an exclusion test or to guide priority of investigations in those with lower gastrointestinal symptoms (chronic diarrhoea) but without rectal bleeding (Grade of evidence level 2, Strength of recommendation weak).

- Faecal calprotectin is recommended to exclude colonic inflammation in those suspected with IBS and under the age of 40 (Grade of evidence level 1, Strength of recommendation strong).

\section{Secondary clinical assessment}

- If symptoms persist despite normal first-line investigations and treatment, then referral for further investigations is recommended (Grade of evidence level 5, Strength of recommendation strong).

- We recommend blood and stool tests to exclude malabsorption and common infections (especially in the immunocompromised or elderly) (Grade of evidence level 2, Strength of recommendation strong).

\section{Common disorders}

- In those with functional bowel or IBS-diarrhoea, a positive diagnosis of bile acid diarrhoea should be made either by ${ }^{75}$ SeHCAT testing or serum bile acid precursor $7 \alpha$-hydroxy-4-cholesten-3-one (depending on local availability) (Grade of evidence level 1, Strength of recommendation strong).

- There is insufficient evidence to recommend use of an empirical trial of treatment for bile acid diarrhoea rather than making a positive diagnosis (Grade of evidence level 5, Strength of recommendation strong).

- We recommend colonoscopy with biopsies of right and left colon (not rectal) to exclude microscopic colitis (Grade of evidence level 1, Strength of recommendation strong).

\section{Malabsorption}

- If lactose maldigestion is suspected, we suggest hydrogen breath testing (if available) or withdrawal of dietary lactose/ carbohydrates from the diet (Grade of evidence level 3, Strength of recommendation weak).

- MR enterography is recommended for evaluation of small bowel abnormalities depending on availability (Grade of evidence level 1, Strength of recommendation strong).

- Video capsule endoscopy (VCE) is recommended for assessing small bowel abnormalities depending on local availability (Grade of evidence level 1 , Strength of recommendation strong).

- We do not recommend small bowel barium follow through or barium enteroclysis for the evaluation of small bowel abnormalities due to its poor sensitivity and specificity (Grade of evidence level 5, Strength of recommendation strong).

- We recommend enteroscopy only for targeted lesions identified by MR enterography or VCE and not for diagnosis of chronic diarrhoea (Grade of evidence level 4, Strength of recommendation strong).

- We recommend faecal elastase testing when fat malabsorption is suspected. We do not recommend PABA testing (Grade of evidence level 1, Strength of recommendation strong).

- MRI (rather than CT) is recommended for assessing structural anomalies of the pancreas in suspected chronic pancreatitis (Grade of evidence level 2, Strength of recommendation strong).

- If small bowel bacterial overgrowth is suspected, we recommend an empirical trial of antibiotics as there is insufficient evidence to recommend routine hydrogen or methane breath testing (Grade of evidence level 3, Strength of recommendation strong).

\section{Surgical and structural disorders}

- We recommend use of anorectal manometry and endoanal ultrasound only when other local pathology has been excluded and conservative measures exhausted (Grade of evidence level 3, Strength of recommendation strong).

- We recommend radiological modalities for the investigation of fistulae-MRI or CT with contrast follow through (Grade of evidence level 3, Strength of recommendation strong).

\section{Rare causes}

- Diarrhoea due to hormone secreting tumours is rare, hence we recommend testing only when other causes of diarrhoea have been excluded (Grade of evidence level 4, Strength of recommendation strong). 
the level of evidence (1-5) and the strength of our recommendation (strong, moderate or weak).

\subsection{Scheduled review of guidelines}

These guidelines will be subject to future revisions within 5 years to take into account new developments and alternative investigative methods.

\subsection{Possible audit goals}

The aim of these guidelines was to establish an optimal investigative scheme for patients presenting with chronic diarrhoea that would maximise a positive diagnosis while minimising the number and invasiveness of investigations. These two potentially opposing directives are influenced by the potential seriousness of the diagnostic outcome. Thus a low threshold for the use of colonoscopy is acceptable in the context of the frequency and clinical significance of colonic neoplasia in older subjects. However, there is less need for extensive investigation where the probability of benign disease is high (eg, in young patients with functional symptoms). ${ }^{4}$ Suggested goals for future audit include:

1. All patients undergoing colonoscopy for chronic diarrhoea should undergo biopsies of the right and left colon for histological examination.

2. Reduction of missed diagnoses of colorectal cancer to $<1 \%$ through utility of faecal immunochemical testing (FIT) and endoscopic examination.

3. Minimising inappropriate first-line investigations (eg, colonoscopy) in patients less than 40 years of age without rectal bleeding and normal faecal calprotectin.

4. Initial non-invasive investigations, including coeliac serology, faecal calprotectin and possibly FIT, should be completed in primary care prior to specialist referral.

5. All patients with persistent undiagnosed chronic diarrhoea should be investigated for bile acid diarrhoea with SeHCAT or serum 7-alpha-hydroxy-4-cholesten-3-one or faecal bile acid measurement where available.

\section{INTRODUCTION}

\subsection{Definition}

Diarrhoea may be defined in terms of stool frequency, consistency, volume or weight. Patients' concept of diarrhoea often focuses around stool consistency. ${ }^{5}$ Indeed, faecal consistency is determined by the water-holding capacity of the stool (ie, the amount of non-bound 'free' water), and this perhaps best defines the concept of diarrhoea. However, quantification of this in clinical practice may prove difficult, hence utility of the Bristol stool chart is recommended-type 5 and above (see online supplementary material). ${ }^{6}$

In the past, stool weights were used ( $\geq 200 \mathrm{~g} /$ day), but this can be misleading as stool weights vary greatly and 'normal' stool volumes can exceed this value, particularly when non-Western diets are encountered. Hence stool weights are not recommended as a measure of diarrhoea.

Further potential for confusion arises from the discrepancy between the medical and 'lay' concepts of diarrhoea, and this needs to be clarified at the initial appraisal. Faecal incontinence in particular is commonly misinterpreted as diarrhoea, ${ }^{7}$ while symptoms relating to functional bowel disease can be difficult to distinguish from organic pathology on the basis of history alone.

There is no consensus on the duration of symptoms that define chronic as opposed to acute diarrhoea. However, most groups including this GDG accept that symptoms persisting for longer than 4 weeks suggest a non-infectious aetiology and merit further investigation. ${ }^{8}$

A recent report suggests that both increased frequency and altered consistency is indicative of organic aetiology. ${ }^{9}$ Thus a pragmatic definition incorporates these elements: chronic diarrhoea is the persistent alteration from the norm with stool consistency between types 5 and 7 on the Bristol stool chart and increased frequency greater than 4 weeks' duration.

\subsection{Prevalence}

Chronic diarrhoea is one of the most common reasons for referral to a gastroenterology clinic. Prevalence rates in Western populations are difficult to estimate, partly through population differences but also through difficulties in definition. In two population surveys, Talley et al reported a prevalence of 'chronic diarrhoea' of between $7 \%$ and $14 \%$ in an elderly population, a proportion of which is likely to include patients with motility disorders (ie, 'functional bowel disease'). Using a definition based on excessive stool frequency without the presence of abdominal pain, estimates of the prevalence of chronic diarrhoea in a Western population are $4-5 \% .{ }^{10}$

\subsection{Difficulties in establishing guidelines for the investigation of chronic diarrhoea}

Reported change in stool frequency or form is characteristic of irritable bowel syndrome (IBS) and indeed forms part of the definition of the condition, which relies on symptom-based criteria and negative physical examination. ${ }^{11}{ }^{12}$ Although stool weight does not usually increase in IBS, as symptom reporting forms the basis for the diagnosis and stool weight is rarely performed early in the course of investigation, considerable overlap between functional bowel disease and true 'diarrhoea' occurs. As IBS may affect $10-13 \%$ of the population, ${ }^{41314}$ there is clearly the potential for inappropriate investigation of patients reporting diarrhoeal symptoms. Conversely, new onset of diarrhoea may reflect serious organic disease such as colonic neoplasia. It is this wide diagnostic potential given similar reported symptoms that makes the introduction of specific guidelines difficult.

The broad range of conditions which lead to diarrhoea also make it difficult to be too prescriptive with regard to the investigative pathways that should be adopted. Diarrhoea may result from: (a) colonic neoplasia; (b) inflammation; (c) small bowel inflammation; (d) small bowel malabsorption; (e) maldigestion due to pancreatic insufficiency; or $(\mathrm{f})$ motility disorders, and it can be difficult to separate these on clinical grounds alone. The decision on whether to focus investigations on any one of these areas remains largely a matter of clinical judgement although, as will be discussed, the prevalence and potential seriousness of certain conditions (eg, colonic neoplasia) necessitates their exclusion early in the investigative scheme.

A further problem in the development of these guidelines has been the large number of investigative methods reported, particularly with regard to malabsorption. This reflects the failure of any single test to become established as the standard and, indeed, many of the available methods have not found a wide acceptance because of inadequate sensitivity, specificity or ease of use. Moreover, there is considerable variation in protocols and analytical methods between laboratories that leads to poor reproducibility of results. ${ }^{15}$ It is also unclear what place some of these tests (some of which were devised prior to the advent of endoscopy) hold in the current investigative scheme that incorporates access to small bowel and colonic histology. To circumvent this somewhat, we propose 
in figure 1 an outline algorithm for the investigation of chronic diarrhoea based on clinical differential diagnosis. This will be used to guide the text in this document.

\section{PRIMARY CLINICAL ASSESSMENT}

The initial assessment of patients with chronic diarrhoea can be mostly carried out in the primary care setting. The importance of careful history taking and examination cannot be overemphasised.

\subsection{Initial presentation in primary care}

In most countries where there is a split primary/secondary care system, the first consultation is with the primary care physician. Around $10 \%$ of all consultations in primary care are for gastroenterological problems, of which half are for lower gastrointestinal (GI) problems. ${ }^{16}$ Most of these are for self-limiting symptoms or for functional GI disorders and only a very small proportion comprise chronic or persistent diarrhoea. Based on the accepted definition of chronic diarrhoea being abnormal passage of $\geq 3$ loose stools per day for more than 4 weeks and a rate of $3-5 \%$ per year, ${ }^{17}$ a primary care physician with an average list size of 1700 patients may have $50-85$ such patients each year. Only a proportion of these will consult, with many managing with self- or pharmacy-based treatments, while others will be managed within primary care, often with antidiarrhoeals.

No data are available on the proportion referred to secondary care, but these are likely to be a small minority of the total number of people with chronic diarrhoea in the community. First-line investigations are normally performed within primary care and patients may be referred if their condition causes interference with normal activities or a compromise of quality of life sufficient to warrant further action. As with other conditions, many patients merely adapt their lives to their symptoms. No formal primary care referral guidelines or recommendations exist for patients with chronic diarrhoea, but the presence of normal first-line investigations with symptoms severe enough to impair quality of life and not responding to treatment constitutes a rationale for referral.

The impact of the symptoms of chronic diarrhoea and the differential diagnoses that need to be considered are clearly different in individual patients. A patient with recent change in bowel habit to include diarrhoea over 6 weeks is likely to need a different approach compared with another patient who has suffered from intermittent watery diarrhoea which has been present for over 5 years. Blood, stool (if an infectious aetiology or if an inflammatory component is suspected) and serological tests (for coeliac disease, hyperthyroidism and anaemia) should be performed in primary care as an initial assessment. Equally, if alarm features (such as unexplained change in bowel habit, persistent blood in the stool and unintentional weight loss) are detected, then referral for further investigations should be initiated.

\subsection{History and examination: secondary care assessment}

A detailed history is essential in the assessment of patients with chronic diarrhoea. This should attempt (a) to establish the likelihood that the symptoms are organic (as opposed to functional) based on presence of 'alarm features' as outlined above; (b) to distinguish malabsorptive from colonic/inflammatory forms of diarrhoea; and (c) to assess for specific causes of diarrhoea.

Symptoms suggestive of an organic disease include a history of diarrhoea of $<3$ months' duration, predominantly nocturnal or continuous (as opposed to intermittent) diarrhoea and significant weight loss. The absence of these, in conjunction with positive symptoms such as those defined in the Rome IV criteria (improved clinical validation $)^{18}$ and a normal physical examination, are suggestive of a functional bowel disturbance, but only with a specificity of approximately $52-74 \% .{ }^{19}$ Unfortunately, these criteria do not reliably exclude inflammatory bowel disease (IBD), microscopic colitis or bile acid diarrhoea, all of which are relatively common and treatable with specific approaches. ${ }^{20}$

Malabsorption is often accompanied by steatorrhoea and the passage of bulky malodorous pale stools. However, milder forms of malabsorption may not result in any reported stool abnormality. Colonic, inflammatory or secretory forms of diarrhoea typically present with liquid loose stools with blood or mucous discharge.

Specific risk factors which increase the likelihood of organic diarrhoea or point to potential lines of investigation should be sought (see box 1). These include:

1. Family history: particularly of neoplastic, inflammatory bowel or coeliac disease.

2. Previous surgery: extensive resections of the ileum and right colon lead to diarrhoea due to lack of absorptive surface and hence fat and carbohydrate malabsorption, decreased transit time or changes in the bile acid pool including malabsorption. ${ }^{21}$ Bacterial overgrowth can often be a problem in this situation, particularly in bypass operations such as in gastric surgery and jejunoileal bypass procedures for morbid obesity. Shorter resections of the terminal ileum can lead to bile acid diarrhoea, which typically occurs after meals and usually responds to fasting and bile acid sequestrants (see sections 5 and 6).

Chronic diarrhoea may also occur in up to $10 \%$ patients after cholecystectomy through mechanisms that include increased gut transit, bile acid diarrhoea, and increased enterohepatic cycling of bile acids. ${ }^{22}$

3. Previous pancreatic disease.

4. Systemic disease: thyrotoxicosis and hypoparathyroid disease, diabetes mellitus, ${ }^{23}$ adrenal disease or systemic sclerosis may predispose to diarrhoea through various mechanisms including endocrine effects, autonomic dysfunction, small bowel bacterial overgrowth or the use of concomitant drug therapy.

5. Alcohol: diarrhoea is common in alcohol abuse. Mechanisms include direct toxic effect on intestinal epithelium, rapid gut transit, decreased activity of intestinal disaccharidases and decreased pancreatic function. ${ }^{24}$

6. Diet: excessive intake of caffeine (eg, coffee, energy drinks), milk in patients with lactase deficiency, food additives (eg, sorbitol), fructose and other FODMAPs (fermentable oligo-, di-, mono-saccharides and polyols) should be sought (see section 5.3).

7. Drugs: up to $4 \%$ of cases of chronic diarrhoea may be due to medications (particularly magnesium supplements, antihypertensives (eg, ACE inhibitors) and non-steroidal anti-inflammatory drugs, newer gliptins (dipeptidyl peptidase-4 inhibitor), theophyllines, antibiotics, antiarrhythmics and antineoplastic agents). ${ }^{25}$

8. Recent overseas travel or other potential sources of infectious gastrointestinal pathogens.

9. Recent antibiotic therapy and Clostridium difficile infection: many different tests are now available for the detection of C. difficile, but most clinical laboratories use a commercial enzyme immunoassay for $C$. difficile toxin (details in section 3.3.3). 
Box 1 Causes of chronic diarrhoea

\section{Common \\ - IBS-diarrhoea \\ - Bile acid diarrhoea \\ - Diet}

- FODMAP malabsorption

- Lactase deficiency is highly prevalent in non-Caucasian ethnic groups

- Artificial sweeteners (eg, sorbitol, xylol in chewing gum, soft drinks)

- Caffeine (eg, coffee, coke, energy drinks)

- Excess alcohol

- Excess liquorice

- Colonic neoplasia

- Inflammatory bowel disease

- Ulcerative colitis

- Crohn's disease

- Microscopic colitis

- Coeliac disease

- Drugs

- Antibiotics, in particular macrolides (eg, erythromycin)

- Non-steroidal anti-inflammatory drugs

- Magnesium-containing products

- Hypoglycaemic agents (eg, metformin, gliptins)

- Antineoplastic agents

- Others (eg, furosemide, Olestra)

Overflow diarrhoea

\section{Infrequent}

- Small bowel bacterial overgrowth

- Mesenteric ischaemia

- Lymphoma

- Surgical causes (eg, small bowel resections, faecal incontinence, internal fistulae)

- Chronic pancreatitis

- Radiation enteropathy

- Pancreatic carcinoma

- Hyperthyroidism

- Diabetes

- Giardiasis (and other chronic infection)

- Cystic fibrosis

Rare

- Other small bowel enteropathies (eg, Whipple's disease, tropical sprue, amyloid, intestinal lymphangiectasia)

- Hypoparathyroidism

- Addison's disease

- Hormone secreting tumours (VIPoma, gastrinoma, carcinoid)

- Autonomic neuropathy

- Factitious diarrhoea

- Brainerd diarrhoea (possible infectious cause not identified)

\subsection{Initial investigations}

\subsubsection{Blood tests}

Abnormal initial screening investigations such as a high erythrocyte sedimentation rate, anaemia or low albumin have a high specificity for the presence of organic disease. ${ }^{26}$ The presence of iron deficiency is a sensitive indicator of small bowel enteropathy, particularly coeliac disease, ${ }^{27}$ but is obviously not a specific test. Guidelines regarding the approach to a patient with iron deficient anaemia have previously been published. ${ }^{28} \mathrm{~A}$ basic screen for evidence of malabsorption should include full blood count, urea and electrolytes, liver function tests, vitamin B12, folate, calcium, ferritin, erythrocyte sedimentation rate and $\mathrm{C}$ reactive protein. Thyroid function tests should also be performed at this stage with a suppressed thyroid-stimulating hormone being the best predictor for hyperthyroidism.

\subsubsection{Serological tests for coeliac disease}

Coeliac disease is defined as a state of heightened immunological responsiveness to ingested gluten in genetically susceptible individuals. ${ }^{29}$ Epidemiological studies screening cohorts of healthy adult volunteers in the USA, UK and other European countries consistently report a prevalence of $0.5-1 \% .^{30-32}$ There is some evidence that the prevalence is increasing, ${ }^{33-35}$ and despite being a common global phenomenon, diagnosis is often delayed. ${ }^{3637}$

The development of highly accurate serological tests and large epidemiological studies have contributed to our current understanding of coeliac disease. It is now recognised that the majority of patients may have more subtle presenting symptoms, adult presentations are more frequent than paediatric, and there is a 'pre-coeliac' state. 323839

Recognition of the coeliac iceberg has improved understanding and detection of coeliac disease. ${ }^{37}$ The visible iceberg above the waterline depicts patients with typical GI symptoms such as diarrhoea and weight loss. The subsequent stratum just below the waterline represents patients considered to have atypical presentations. They may have vague, non-specific GI symptoms such as bloating, or conditions associated with coeliac disease such as iron deficiency anaemia, osteoporosis and persistently abnormal liver function tests. ${ }^{40-42}$ Among certain groups the prevalence of coeliac disease will be higher (eg, type 1 diabetes, autoimmune thyroid disease or a first-degree relative with coeliac disease). ${ }^{43}$

Diarrhoea may be present in $43-85 \%$ of patients with newly diagnosed coeliac disease. ${ }^{40-42} 4445$ Conversely, the prevalence of coeliac disease in patients referred to secondary care with chronic diarrhoea has been reported to range from $3 \%$ to $10 \% .{ }^{46}{ }^{47}$ Given the delays in diagnosis for patients with undetected coeliac disease and the fact that the tests are inexpensive ${ }^{43}$ suggests that serological testing for coeliac disease in patients presenting with chronic diarrhoea is mandatory.

Endomysial antibody (EMA) and tissue transglutaminase antibody (TTG IgA) have a combined sensitivity and specificity of over $90 \%$ when used in combination in selected populations with a high prevalence of coeliac disease. ${ }^{48} 49$ However, when the prevalence of coeliac disease falls to $1 \%$, as found in screening populations, the positive predictive value of these tests falls to around $80 \%$ or less. ${ }^{48} 49$ The sensitivity of the serological tests also falls well below $90 \%$ when histological grades less than Marsh 3 (villous atrophy) are considered. ${ }^{48-52}$ An IgA immunoglobulin level is also necessary as both EMA and TTG are IgA-based. In the presence of IgA deficiency, IgG EMA or IgG TTG should be performed. ${ }^{53}$ Finally, if the clinician is still suspicious, then a duodenal biopsy should be performed even in the presence of negative antibodies. Antibody-negative coeliac disease accounts for $6.4-7 \%$ of cases of patients presenting with coeliac disease. ${ }^{485}$

\subsubsection{Immunodeficiency and infections}

Immunodeficiency states complicate diagnosing the cause of diarrhoea. The first step is to identify the immunodeficiency. Beyond the common primary and haematological causes, clinicians should be aware that chronic diarrhoea is a common symptom in patients newly diagnosed with human immunodeficiency virus 
(HIV). ${ }^{54}$ Clinicians should be aware of HIV prevalence estimates in their region and test accordingly. Once an immunodeficiency has been identified, potential chronic infection with pathogens such as cryptosporidia or norovirus should be investigated.

Chronic diarrhoea due to infectious agents is unusual in the immunocompetent patient. Protozoan infections, such as giardiasis and amoebiasis, are most likely to result in chronic infections. Examination of three fresh stools for ova, cysts and parasites remains the mainstay of diagnosis and has a sensitivity of approximately $60-90 \%$ for detection of these organisms. If there is doubt about persisting Giardia infection, then the use of a stool ELISA (92\% sensitivity and 98\% specificity) has largely replaced the need for intestinal biopsies and wet (saline and iodine mount) preparations. ${ }^{55-58}$ Short course treatment with metronidazole or tinidazole is effective. ${ }^{58}$ Serological testing (indirect haemagglutination test or ELISA) can be a useful adjunct in cases of amoebic liver abscess-more so in endemic areas. ${ }^{59}$ Clinicians should liaise with their local microbiology services to establish the diagnostic methods used, which may include transition to newer ELISA-based assays with greater sensitivity and specificity.

A notable exception is Clostridium difficile infection where diarrhoea can persist through failure of initial treatment or rapid relapse, which has consistently occurred in one in four patients in the placebo arms of recent clinical trials. ${ }^{60-62}$ Identification of a wide variation in testing approaches across Europe has led to recommendations for a standardised two-stage approach. ${ }^{63}$ The first step identifies the presence of the organism with glutamate dehydrogenase enzyme immunoassay (EIA), nucleic acid amplification testing or PCR. The second stage looks to demonstrate active $C$. difficile toxin production through toxin EIA. Such a combination of a sensitive and then a specific test gives high negative and positive predictive values where tests agree. Where they do not, clinical judgement on the likelihood of infection will determine the need for treatment. The phenomenon of post-infectious IBS after C. difficile infection is recognised, ${ }^{64}$ hence clinicians should be wary of excessive antimicrobial therapy on the basis of PCR alone, especially in the absence of clear evidence of toxin production.

Recommendations

- We recommend an initial screening blood test (full blood count, ferritin, tissue transglutaminase/EMA and thyroid function test) as well as stool tests for inflammation (faecal calprotectin) should be undertaken in primary care (Grade of evidence level 3, Strength of recommendation strong).

- We recommend screening for coeliac disease using serological tests (tissue transglutaminase or EMA), which have a high sensitivity and specificity for the disease (Grade of evidence level 1, Strength of recommendation strong).

- If IgA deficient, we recommend either IgG EMA or IgG TTG should be performed (Grade of evidence level 2, Strength of recommendation strong).

- HIV infection should be excluded in those who are immunocompromised and present with chronic diarrhoea (Grade of evidence level 2, Strength of recommendation strong).

- We recommend combination testing for Clostridium difficile infection; confirmation of the presence of the organism by glutamate dehydrogenase enzyme immnunoassay or PCR and determining if these are toxin-producing (toxin EIA) (Grade of evidence level 2, Strength of recommendation strong).

\section{CANCER OR INFLAMMATION}

\subsection{Stool tests}

The Bristol stool chart (see online supplementary material), which is validated, is a good tool to determine stool consistency and guide subsequent investigations. ${ }^{65}$ Stool weights are no longer used or recommended due their limited clinical value.

\subsubsection{Faecal calprotectin}

The inflammatory marker faecal calprotectin has recently been shown to be of value and the subject of a NICE Diagnostic Guideline (DG11). ${ }^{66}$ Calprotectin is released when inflammatory processes occur due to the degranulation of neutrophil granulocytes. When the inflammation is within the intestinal tract, calprotectin is released into the intestinal lumen and is stable enough to be measured in faeces. NICE recommends the use of faecal calprotectin as an option in the differential diagnosis of IBS and IBD in adults with recent onset lower GI symptoms in whom cancer is not suspected. Thus patients with low calprotectin levels are unlikely to have any active inflammatory processes at the time of sample collection, making a diagnosis of IBD less likely. ${ }^{6768}$ NICE uses the commonly quoted $50 \mu \mathrm{g} / \mathrm{g}$ faeces as the decision level above which IBD is more likely, although other causes of raised calprotectin include colorectal cancer, infectious gastroenteritis and non-steroidal anti-inflammatory drugs. ${ }^{69}$ This strategy should lead to a reduced level of secondary care referrals with associated lower cost and morbidity. There is some evidence that calprotectin levels $>250 \mu \mathrm{g} / \mathrm{g}$ faeces suggest active inflammation correlating quite well with endoscopic inflammation. ${ }^{67} 70$ The issue of indeterminate levels still remains elusive, although one study that looked at the 12-month outcome of indeterminate faecal calprotectin levels $(50-249 \mu \mathrm{g} / \mathrm{g}$ faeces) noted an $8 \%$ chance of developing IBD compared with $1 \%$ in those with levels $<50 \mu \mathrm{g} / \mathrm{g}$ faeces. ${ }^{71}$ For further details see online supplementary material.

\subsubsection{Faecal occult blood test (FOBT)/faecal immunochemical technique (FIT)}

The detection of excess blood in faeces has been used for many years in the diagnosis of colorectal cancer. There are two types of faecal occult blood test: the older gFOBT based on the oxidisation of guaiac gum and the newer FIT for the detection of faecal haemoglobin, which uses an immunochemical technique to detect blood. Current European guidelines for colorectal cancer screening ${ }^{72}$ recommend the use of faecal haemoglobin and, although the UK still uses the older test, plans are well advanced to change to faecal haemoglobin in both Scotland and England in 2018 for screening. Emerging studies indicate that, in those with lower GI symptoms suggestive of colorectal cancer, FIT testing has a high negative predictive value $(0.99)$ with the optimal cut-off between 7 and $10 \mu \mathrm{g} / \mathrm{g}$ faeces. ${ }^{73-75}$ With the appropriate quality assurance processes in place and good clinical judgement, there is good evidence that using faecal haemoglobin in symptomatic patients may be of benefit in reducing the burden of unnecessary colonoscopic examinations. For further details see online supplementary material.

\subsection{Endoscopic and histological assessment}

In most patients with chronic diarrhoea, some form of endoscopic investigation will be necessary. In young patients (less than 40 years) reporting 'diarrhoea' but who have other typical symptoms of a functional bowel disorder and negative initial investigations, a diagnosis of IBS may be made in the primary care setting without recourse to further investigations. ${ }^{76}$ There 
is some evidence that using anti-vinculin and anti-cytolethal distending toxin $\mathrm{B}$ antibodies may help with making a positive diagnosis of IBS. ${ }^{77}$ However, patients under 40 years without typical symptoms of functional bowel disorder and/or severe symptoms and documented diarrhoea (as previously defined) should have further evaluation (see section 3.2.3).

\subsubsection{Colonoscopy}

Screening colonoscopy in asymptomatic individuals detects colonic adenomas in $14.4-37.5 \%$ of cases ${ }^{57}$ but few studies have addressed the frequency of neoplasia in symptomatic patients, and none has specifically addressed the prevalence of adenomas in patients undergoing colonoscopy for diarrhoea. Neugut and colleagues ${ }^{78}$ showed that, of 861 patients (with rectal bleeding), only 154 had a change in bowel habit of which 7\% of these had colonic neoplasms. This would suggest that change in bowel habit is a poor guide to the prevalence of neoplasia unless advanced to the point of malignancy. Almost half had neoplasia proximal to the splenic flexure, indicating the need for full colonoscopy rather than flexible sigmoidoscopy in these patients. ${ }^{789}$

In addition to neoplasia, colonoscopy also has a diagnostic yield for other conditions ranging from $7 \%$ to $31 \%$, with IBD and microscopic colitis being most commonly found. Hence both right- and left-sided colonic biopsies are necessary (see further details in section 5.2).$^{80-83}$ Routine ileoscopy further adds to the value of colonoscopy. This led to a positive diagnosis in $18 \%$ of non-HIV patients who complained of diarrhoea. ${ }^{84}$ In patients in whom IBD is suspected, the value of ileoscopy and biopsy is further enhanced: $36 \%$ of patients with a normal colonoscopy and diarrhoea had terminal ileal disease. ${ }^{85}$ Although these results clearly reflect considerable referral bias, when taken together they suggest that, in chronic diarrhoea of uncertain origin, colonoscopy and ileoscopy with biopsy will lead to a diagnosis in approximately $15-20 \%$ of cases.

Colonoscopy is more sensitive than barium enema and, given this and the need to obtain histology to exclude colitis, barium studies of the colon are no longer indicated in chronic diarrhoea. ${ }^{8687}$

\subsubsection{Flexible sigmoidoscopy}

Unprepared rigid sigmoidoscopy has long been used in the outpatient setting to quickly assess the rectum and stool. This remains an appropriate examination in those younger patients who on clinical grounds are believed to have a functional bowel disorder. Some authors conclude that, in this age group, most pathology occurs in the distal colon and is thus accessible with a flexible sigmoidoscope. ${ }^{8}{ }^{80}$ In one study that examined the prevalence and anatomical distribution of colonic pathology in patients presenting with non-HIV-related chronic diarrhoea, $15 \%$ of patients had colonic pathology. ${ }^{88}$ Some $99 \%$ of these diagnoses could have been made from biopsies of the distal colon using a flexible sigmoidoscope, the primary diagnoses being microscopic colitis, Crohn's disease and ulcerative colitis. Other sources point more strongly to the need for full colonoscopy in chronic diarrhoea of uncertain aetiology. ${ }^{57-62}$

\subsubsection{Upper gastrointestinal endoscopy}

There is little information on the diagnostic yield of upper GI endoscopy in patients whose diarrhoea is suspected to be due to malabsorption. This will clearly vary depending on the cohort of patients being investigated, referral criteria and degree of suspicion for any given underlying diagnosis. Coeliac disease should be considered and investigated as per section 3.3.2.

\subsection{Small bowel imaging and visualisation for inflammation} 4.3.1 Small bowel imaging for abnormality (eg, inflammatory bowel disease)

Traditionally the small bowel barium follow through (SBBFT) or barium enteroclysis was the standard means of assessing small bowel mucosa. However, barium examinations have both a low sensitivity and specificity in the detection of small bowel abnormalities. ${ }^{89}$ Ultrasonography (USS) may be used to assess the bowel, and the advantages include its non-invasiveness and availability. USS has been shown to have high sensitivity in the detection of terminal ileal pathology but its accuracy for disease proximal to the terminal ileum is lower compared with CT and MRI. ${ }^{90-92}$ The significant disadvantages of USS include the difficulty of viewing the GI tract in its entirety and high operator dependence. ${ }^{93-95}$

Cross-sectional modalities such as CT and MRI have emerged as the preferred imaging tests for evaluation of the small bowel. Specialised small bowel imaging procedures such as CT and MR enterography and enteroclysis have become preferred, as comparative studies have shown their superiority compared with SBBFT. $^{93} 95$

The enterographic technique (CT or MR) is based on imaging after ingestion of a large amount of oral contrast over a set time period whereas the enteroclysis procedure requires nasojejunal intubation. The main disadvantage of an enteroclysis examination is the nasojejunal intubation, which is uncomfortable for patients leading to greater patient preference for enterography examinations. 9697

Recent validated studies have reported high accuracy of MR enterography examination for small bowel abnormalities compared with surgical findings. ${ }^{94}{ }^{98-101}$ Further advances in MR technology with diffusion-weighted imaging (MR- DWI) are proving important for the diagnosis and assessment of bowel abnormalities. ${ }^{102} 103$ Although CT is also widely used in abdominal imaging, its main disadvantage is the associated high radiation burden. A recent meta-analysis has concluded that it is preferable to use a non-ionising modality such as USS or MRI in order to reduce radiation exposure. ${ }^{98} 104$ Several comparative studies also report that MRI has higher sensitivity than CT in the detection of small bowel diseases and neoplasms. ${ }^{949698-100103105}$

Current evidence-based analysis indicates that either CT or MR enterography (depending on availability) should be the preferred initial test for the diagnosis of small intestinal abnormalities in patients with chronic diarrhoea. MRI is the preferred option as it does not entail high-dose radiation compared with CT imaging. ${ }^{101} 105$

\subsubsection{Video capsule endoscopy}

Capsule endoscopy has a role either as a means to distinguish small bowel abnormalities or to assess further the small bowel after a negative radiological (MR or CT) investigation. However, in some centres this may be viewed as a first-line investigation of the small bowel due to its non-invasive nature (patient tolerability and acceptance) as well as increased diagnostic yield when compared by meta-analysis and systematic review against other small bowel investigations. ${ }^{106}$

When specifically considering coeliac disease, capsule endoscopy is not recommended and patients should be encouraged to have a duodenal biopsy in order to obtain a histological diagnosis. For patients who are unable or unwilling to undergo a gastroscopy, a small bowel capsule may help to demonstrate villous atrophy in the presence of positive coeliac serology. 


\subsubsection{Enteroscopy}

Small bowel enteroscopy has been evaluated as a complementary investigation to SBBFT and capsule endoscopy, either as a means to distinguish small bowel abnormalities or to assess further the small bowel after a negative radiological investigation. ${ }^{107}$ A key feature of enteroscopy studies in assessing inflammation or obscure bleeding is the consistently high false negative rate of prior upper and lower endoscopy, emphasising the need to ensure that adequate visualisation and biopsy of the duodenum and ileum have been achieved. Push enteroscopy may have some value to obtain jejunal biopsies in determining rare causes of diarrhoea-for example, cyclospora infections, sprue-related strongyloidosis or those with malabsorption/raised IgA TTG and non-diagnostic duodenal biopsies. ${ }^{108}$ Device-assisted enteroscopy

\section{Recommendations}

- We recommend use of faecal calprotectin in younger patients (under 40 years) with chronic diarrhoea in whom inflammation is suspected and not cancer (Grade of evidence level 1, Strength of recommendation strong).

- Cut-off of $50 \mu \mathrm{g} / \mathrm{g}$ faeces (assay-dependent) is recommended to distinguish functional bowel disorder from organicl inflammatory bowel disease (Grade of evidence level 1, Strength of recommendation strong).

- In patients with typical symptoms of functional bowel disease, normal physical examination and normal screening blood and faecal tests (calprotectin), a positive diagnosis of IBS can be made (Grade of evidence level 2, Strength of recommendation strong).

- For those with lower gastrointestinal symptoms suspicious of colon cancer (without rectal bleeding), we suggest faecal immunological testing as a rule out test to guide need for referral or urgency of investigations either in primary or secondary care; (Grade of evidence level 2, Strength of recommendation weak)

- In patients with chronic diarrhoea, we recommend colonoscopy (with ileoscopy) and biopsy as the preferred investigation of the lower bowel (Grade of evidence level 1, Strength of recommendation strong).

- In younger patients (under 40 years) with a normal faecal calprotectin and in whom functional bowel disease is suspected, we recommend a flexible sigmoidoscopy with biopsy (Grade of evidence level 3, Strength of recommendation strong).

- We recommend either MR enterography or video capsule endoscopy (VCE) (depending on local availability) rather than CT as first-line investigation for diagnosing inflammation within the small bowel (Grade of evidence level 1, Strength of recommendation strong).

- We do not recommend VCE for the diagnosis of coeliac disease due to insufficient evidence (Grade of evidence level 5 , Strength of recommendation strong).

- Ultrasonography of the small bowel, while attractive due to its non-invasive nature and absence of radiation exposure, has a limited diagnostic role hence cannot be recommended routinely (unless other modalities are unavailable) (Grade of evidence level 4, Strength of recommendation strong).

- Enteroscopy ( \pm device assisted) has limited diagnostic value for chronic diarrhoea and we recommend its role mainly for targeting predefined lesions (Grade of evidence level 4, Strength of recommendation strong). (double balloon, single balloon or spiral) is complementary to capsule endoscopy and should be reserved for targeting lesions (and obtaining histology) or therapeutic intervention when abnormalities are identified by small bowel imaging or capsule endoscopy. ${ }^{106}$

\section{COMMON DISODERS}

\subsection{Bile acid diarrhoea}

Given that a third of patients labelled with diarrhoea-predominant IBS actually suffer from bile acid diarrhoea, this condition is more common than initially perceived with a significant impact on patients. ${ }^{109110}$ Bile acids are required for the digestion of fat and are synthesised from cholesterol in the liver and excreted in the bile mainly in the form of glycine or taurine conjugates. This process is regulated through a negative feedback manner by nuclear farnesoid receptor as well as by fibroblast growth factor 19 (FGF-19), ${ }^{111} 112$ a protein released from ileal enterocytes. Up to $95 \%$ of bile acids are reabsorbed through the enterohepatic circulation.

${ }^{75}$ SeHCAT testing (the standard radiolabelled test used to identify bile acid diarrhoea: tauroselcholic (Se) acid) was first described in $1982 .{ }^{113} 114$ A retention of $10-15 \%$ at 7 days is usually defined as mild bile acid loss, $5-10 \%$ as moderate and $0-5 \%$ as severely abnormal. These values also predict response to therapy with bile acid sequestrants. ${ }^{115}$

The serum bile acid precursor $7 \alpha$-hydroxy-4-cholesten3 -one ${ }^{116}$ is an intermediary product in the synthesis of bile acids from cholesterol by the liver enzyme CYP7A1 and so provides a measure of bile acid synthesis. It has a negative predictive value of 95\% (positive predictive value of 74\%) compared with SeHCAT, hence making it attractive as a screening test for excessive bile acid turnover. ${ }^{117}$ Levels above $47.1 \mathrm{ng} / \mathrm{mL}$ are indicative of bile acid diarrhoea. ${ }^{118}$ C4 requires a fasting sample, like FGF-19; both undergo diurnal and postprandial variation, with false positives also occurring in those with liver disease. ${ }^{119}$

Faecal bile acid measurement is another option with values $>2300 \mu \mathrm{mol} / 48$ hours indicative of bile acid diarrhoea. ${ }^{119}$ However, as dietary fat intake and consequently bile acid levels are variable, it requires a 48-hour sample. Moreover, it is cumbersome and not yet commercially available in the UK. Additionally, other less invasive and economical tests such as urine testing for 2-propanol and acetone are currently being evaluated. ${ }^{120}$ A summary table of tests in bile acid diarrhoea are presented in these studies. ${ }^{121} 122$

Up to $30 \%$ of patients with diarrhoea-predominant IBS have evidence of bile acid diarrhoea as determined by SeHCAT testing. ${ }^{87} 109123$ Compared with controls, IBS patients had lower SeHCAT values and higher C4 levels but similar FGF-19 levels. More than 50\% responded to bile acid sequestrants (colestipol). ${ }^{124}$ In addition to patients with ileal disease (eg, Crohn's disease), ${ }^{125}$ bile acid diarrhoea has also been reported in patients following cholecystectomy and post-infectious diarrhoea. ${ }^{126}$ For those not responding to treatment, other additional causes should be sought (eg, bacterial overgrowth, pancreatic insufficiency or microscopic colitis), ${ }^{127}$ even if SeHCAT testing has been abnormal. Another under-recognised group are those with cancer who have received pelvic chemoradiotherapy as $>50 \%$ have bile acid diarrhoea. ${ }^{128}$

The NICE DG7 diagnostic guidance report on SeHCAT in 2013 and subsequent update in 2016 recognised the potential for patient and system benefits associated with SeHCAT investigations. ${ }^{129}$ One study suggests that the economic evaluation needs to be reconsidered as the impact of not making a positive 
diagnosis leads to repeat (unnecessary) testing with additional cost to the NHS. ${ }^{130}$ More recently it has been shown that making a positive diagnosis of bile acid diarrhoea (in this study using SeHCAT) was economically beneficial as those who had negative SeHCAT tests underwent significantly more investigations $(1.8 \mathrm{x}$ more), especially cross-sectional imaging $(13 \mathrm{x}$ more likely), although those with a positive SeHCAT had more clinical appointments. ${ }^{131}$

A systematic review and meta-analysis comprising 36 studies and 5028 patients on bile acid diarrhoea biomarkers concluded that SeHCAT had the highest diagnostic yield to date (limited by study heterogeneity), with $25 \%$ previously diagnosed as having functional diarrhoea actually having primary bile acid diarrhoea. $^{122}$

In a series of 264 patients where $53 \%$ had bile acid diarrhoea, $44 \%$ failed to respond to cholestyramine alone. Half of these non-responders derived benefit from colesevelam (unlicensed but used with extended indication). Thus, lack of response to cholestyramine does not constitute exclusion of bile acid diarrhoea, ${ }^{132}$ hence therapeutic trials of bile acid sequestrants (cholestyramine or colesevelam) are not recommended. Pooled data from 15 studies show a dose-response relationship between the severity of malabsorption and the effect of treatment with a bile acid sequestrant: clinical response to cholestyramine occurred in $96 \%$ of patients with $<5 \%$ retention of SeHCAT, $80 \%$ at $<10 \%$ retention and $70 \%$ at $<15 \%$ retention. ${ }^{115}$

There are also logistic considerations for high throughput nuclear medicine departments as other concurrent studies may result in background radiation which could affect the 7-day SeHCAT retention value; individual departmental protocols should be in place to circumvent this issue. ${ }^{87}$

Bile acid diarrhoea should no longer be missed given the available option to test with either SeHCAT or serum C4. Other emerging serum and urine tests may soon be available. Once diagnosed, treatment is simple and effective.

\subsection{Microscopic colitis}

Collagenous colitis and lymphocytic colitis are two forms of microscopic colitis, both commonly presenting with chronic, non-bloody watery diarrhoea and with few or no endoscopic abnormalities. ${ }^{133}$ They were first described around 1980. ${ }^{134}{ }^{135}$ Histological features are distinct from ulcerative colitis or Crohn's disease and are not reliably detected on macroscopic examination at colonoscopy. Microscopic examination shows an increased number of intraepithelial and lamina propria lymphocytes ( $>20 / 100$ cells) in both forms of microscopic colitis, together with a thickened subepithelial collagen band $(>10 \mu \mathrm{m})$ in collagenous colitis. ${ }^{136}$

These conditions have been the subject of several recent systematic reviews and meta-analyses and data from these have been used to inform the current guidance. The clinical presentation, risk factors, pathology, disease course and response to treatment have been reviewed and compared for lymphocytic and collagenous colitis. ${ }^{137} 138$ The epidemiology and trends ${ }^{139}$ and the diagnostic overlaps with IBS and functional GI disease have been reviewed. ${ }^{140141}$ The American Gastroenterology Association has developed guidelines on the medical management of microscopic colitis. ${ }^{142} 143$

The overall prevalence of microscopic colitis ranges from about 50 to 200/100 000. ${ }^{143}$ Both forms are commoner in women (collagenous colitis 77\%, lymphocytic colitis 68\%) and the mean age at presentation is around $60 .{ }^{138}$ However, microscopic colitis can present in much younger patients with $25 \%$ of cases being under $45 .^{137}$ The incidence of collagenous and lymphocytic colitis has been increasing in parallel, possibly related to greater awareness of the need to look for this diagnosis. There are overlapping histological features and the two forms cannot be distinguished on the basis of symptoms, although they may be less severe in lymphocytic compared with collagenous colitis. ${ }^{133}$ Nocturnal diarrhoea and incontinence are frequently present.

In a meta-analysis of studies of patients meeting the criteria for diarrhoea-dominant IBS, the prevalence of microscopic colitis was $9.8 \%(95 \%$ CI $4.4 \%$ to $17.1 \%) .{ }^{140}$ Other reviews have also quoted a prevalence in patients being investigated for chronic diarrhoea of around 7.5-10\%. ${ }^{133} 142$ Other functional bowel disorders, including constipation-predominant or mixed IBS, may also coexist with microscopic colitis. In patients with a diagnosis of microscopic colitis, about one-third have symptoms compatible with IBS, but this is a similar proportion to patients with other causes of chronic diarrhoea. ${ }^{141}$

Conditions associated with microscopic colitis include autoimmune diseases such as rheumatic disease, thyroid disease and coeliac disease (in around 5-7\%). ${ }^{133} 137142$ Bile acid diarrhoea (diagnosed by SeHCAT testing) has been reported to be prevalent in both collagenous colitis $(41 \% ; 37-45 \%)$ and lymphocytic colitis $(29 \% ; 24-34 \%) .{ }^{138}$ Use of non-steroidal anti-inflammatory drugs, proton pump inhibitors and sertraline is also high and withdrawal of these can be associated with improvement in symptoms. ${ }^{138}$

Diagnosis of microscopic colitis is made by histological examination of colonic mucosal biopsies. Multiple biopsies should be taken and examined to detect the histological changes of lymphocyte infiltration and a possible collagenous subepithelial band. ${ }^{136}$ It needs to be stressed that the colonoscopic macroscopic appearances may be essentially normal, but biopsies are still needed. Right-sided biopsies, taken at colonoscopy, have a greater yield than rectal biopsies, but most patients will have changes detectable in the distal colon and so can be diagnosed by biopsies taken at flexible sigmoidoscopy. ${ }^{82} 83$ There is no reliable biomarker for microscopic colitis, although a raised faecal calprotectin may be found, ${ }^{144}$ which will suggest the need for further colonoscopic assessment. A scoring system has been developed which has a good negative predictive value $(97 \%)$ to exclude microscopic colitis. ${ }^{145}$ This may have a role in avoiding unnecessary colonoscopic biopsies in some patients.

Making the diagnosis of microscopic colitis in a patient with chronic diarrhoea is important as specific and effective treatment for the condition is now available. Budesonide, in controlled release preparations, has been shown to induce remission in active disease for both forms of microscopic colitis, and these trials have recently been reviewed. ${ }^{143}$ There is also good evidence for budesonide in the maintenance of remission in collagenous colitis. ${ }^{146}$ Up to $70 \%$ can relapse and require further treatment, ${ }^{147}$ but others can remain symptom-free. Other drugs have been used, including prednisolone, bile acid sequestrants in appropriate patients with demonstrated bile acid diarrhoea and, in steroid-refractory patients, immunosuppressives. ${ }^{142}$

\subsection{Maldigestion of fructose-based carbohydrates and lactose and polyhydric alcohols}

Maldigestion of fermentable oligo-, di-, mono-saccharides and polyols (FODMAPs) is a cause of diarrhoea in patients with IBS and other patient groups ${ }^{148}$ (eg, IBD in remission, enteral nutrition). The mechanism by which these nutrients have effects on the GI tract has been described through detailed physiological and imaging studies. ${ }^{149-152}$ 
Fructose is a hexose monosaccharide that is found in food on its own, as a disaccharide with glucose-forming sucrose and as longer chain polymers called fructans. Fructose is usually absorbed at the epithelium via the facilitative transporter GLUT5 and the GLUT2 glucose-fructose co-transporter. ${ }^{153}$ The modern diet may contain high levels of isolated fructose or fructose in excess of glucose that exceed the absorption capacity of the small bowel (eg, soft drinks sweetened with corn syrup). Undigested fructose then passes into the colon and is fermented in the same manner as lactose in patients who are deficient in small intestinal lactase due to non-persistence of this enzyme expression into adult life.

Fructans are present in large concentrations in wheat, a staple of the diet for many populations (eg, bread, noodles). Fermentation of fructans by colonic bacteria is the cause of 'wheat intolerance' in many patients without coeliac disease. ${ }^{154} 155$ Similarly, sorbitol and other non-absorbed sugar alcohols ('polyols') used as artificial sweeteners pass unchanged into the colon and can induce diarrhoea if taken in large quantities (eg, diet drinks, chewing gum).

Interpretation of carbohydrate breath tests is particularly contingent on the challenge dose used. Double-blind randomised controlled dietary challenge studies show a dose-response relationship between the intake of lactose, fructose and fructans with the likelihood of malabsorption (as assessed by an increase in breath hydrogen) and the development of abdominal symptoms, including diarrhoea. ${ }^{150}$ The prevalence of primary lactase deficiency based on the presence of the $13910 \mathrm{C} / \mathrm{C}$ genotype in the promoter region of the lactase enzyme gene is $2-15 \%$, predominantly in Caucasian populations in Northern Europe. ${ }^{156}$ A recent meta-analysis of epidemiological data reported that $68 \%$ (95\% CI 64\% to $72 \%$ ) of the world's population are lactase-deficient, ${ }^{157}$ and this approaches $100 \%$ in the Han Chinese population. ${ }^{158}$ There is a high level of concordance between the presence of this genotype and lactose malabsorption. ${ }^{156}$ In contrast, there is no unique genotype linked with fructose or fructan malabsorption and a review of nine studies found that $25 \mathrm{~g}$ fructose may induce an increase in breath hydrogen in $40 \%$ of healthy subjects, but rarely induces symptoms. ${ }^{153}$ Prospective studies have shown that self-reported dietary intolerance is a poor predictor of objective test results ${ }^{158159}$ or even response to dietary restriction. Thus, breath testing after carbohydrate challenge could be useful if it identifies the cause of symptoms and predicts response to therapy. A positive lactose breath test fulfils these criteria, ${ }^{160} 161$ although avoidance of dairy products alone is rarely sufficient treatment in patients with IBS. ${ }^{161} 162$ No trials have directly addressed patients with isolated diarrhoea but, in diarrhoea-predominant IBS, two randomised controlled trials ${ }^{163164}$ and one prospective study ${ }^{165}$ have found no relationship between the result of breath testing and response to dietary fructose reduction. At present, fructose breath testing cannot be said to inform diagnosis and treatment of fructose intolerance.

\subsection{Post radiation diarrhoea}

This is increasingly more common given that there is a threefold rise in cancer survivors in the last three decades. Many of the causes of chronic diarrhoea discussed in other sections of these guidelines are common in people who have been treated for GI and other cancers (eg, bile acid diarrhoea, pancreatic insufficiency). Specific guidance is now available as to how to investigate such patients, especially for the late effects of radiation therapy. ${ }^{166}$

\subsection{Rapid intestinal transit}

Many conditions associated with diarrhoea have been ascribed to abnormalities of gut motility and increased intestinal transit time. These include post-surgical states (eg, vagotomy), endocrine conditions (eg, hyperthyroidism), bile acid diarrhoea, autonomic neuropathy (eg, diabetes) and, most often, primary functional diarrhoea. Assessment of the contribution of disordered motility and transit to diarrhoeal disease is hampered by the fact that (a) many of these conditions have multifactorial aetiologies; (b) available tests have limited ability to identify the cause of symptoms; and (c) wide individual variation in normal values precludes a definitive diagnosis in all except the most severe cases.

Detailed measurements of motility can be acquired by small intestinal or colonic manometry, ideally with high-resolution technology that clearly visualises propulsive and retrograde contractions that promote and inhibit transit. ${ }^{167}$ Pressure measurements are abnormal in the presence of obstruction and gross neuromuscular dysfunction, but findings in patients with severe symptoms do not correlate well with pathology on small bowel biopsy. ${ }^{168}$ In patients with chronic diarrhoea and IBS, the results are almost always normal and such invasive techniques are not well tolerated. ${ }^{169} 170$

\section{Recommendations}

- If functional bowel disease or IBS-diarrhoea is suspected, we recommend that bile acid diarrhoea should be excluded with either ${ }^{75}$ SeHCAT testing or fasting serum C4 ( $7 \alpha$ hydroxy4-cholesten-3-one) levels. A test and treat approach is recommended as opposed to blind empirical therapy unless no diagnostic test is available (Grade of evidence level 1, Strength of recommendation strong).

- Patients with very low ${ }^{75}$ SeHCAT values are most likely to have a response to treatment with bile acid sequestrants. We recommend this is tried if the ${ }^{75} \mathrm{SeHCAT}$ value is $<15 \%$ or the fasting serum $\mathrm{C} 4$ is raised above defined laboratory values (Grade of evidence level 2, Strength of recommendation strong).

-We recommend colonic biopsies to exclude microscopic colitis. These should be at least from the left side (not rectal) and, as microscopic colitis can be patchy, right-side biopsies may improve diagnostic yield (Grade of evidence level 1, Strength of recommendation strong).

- Both glucose and lactulose hydrogen breath tests have poor sensitivity and specificity and are not recommended for the diagnosis of small bowel bacterial overgrowth (Grade of evidence level 2, Strength of recommendation strong).

- The utility of concurrent hydrogen and methane breath testing with measurements of orocaecal transit time could reduce a false positive diagnosis due to variability in intestinal transit, but has not been subject to definitive clinical study hence cannot be recommended (Grade of evidence level 4, Strength of recommendation strong).

- We recommend investigating for intestinal transit with manometry only if there is clinical suspicion and failure to respond to therapy (Grade of evidence level 4, Strength of recommendation strong). 
Only patients who remain undiagnosed after extensive traditional investigation and failed medical therapy should be considered for manometry studies. ${ }^{171}$ In the future non-invasive MRI may find a role in the diagnosis of diarrhoeal conditions. Recent studies have shown that this technique can provide comprehensive measurements of both motility and transit and can identify specific abnormalities in patients with diarrhoea-predominant IBS. ${ }^{172}$

\section{TESTS FOR MALABSORPTION}

Malabsorption may occur as a result of a defective luminal environment such as inadequate bile acid or enzyme function, failure of epithelial transport due to mucosal disease or structural disorders (eg, fistula, surgical resection). Although there are generally coexisting deficiencies of fat, carbohydrate, protein, vitamins and minerals, the effects of one of these may predominate. Thus, pancreatic exocrine insufficiency is the most frequent cause of severe predominant steatorrhoea where faecal fat excretion exceeds $13 \mathrm{~g} /$ day $(47 \mathrm{mmol} /$ day $) .{ }^{173}$ This is rare in mucosal or structural disease, although milder forms of steatorrhoea are common. In comparison, carbohydrate malabsorption is predominantly associated with mucosal disease or dysfunction. Approaches to investigation involve either detection of the relevant compound in faeces or detection of an absorbed substance in the blood, urine or other tissues. A variation of the latter is the use of breath testing, which relies on the breakdown and bacterial fermentation of the test substance. These investigations (stool fat, isotope breath or faecal tests, sugar and other permeability tests) were detailed by a previous version of these guidelines ${ }^{174}$ and will not be covered further here because, with the exception of hydrogen breath tests (see section 6.5.2), none have received significant support in publications since 2003 and they have not established themselves in clinical practice outside specialist centres.

\subsection{Fat malabsorption}

Pancreatic exocrine insufficiency can lead to malabsorption (or more correctly maldigestion) and diarrhoea due to impairment of pancreatic enzyme and bicarbonate secretion. In adults, this is usually the result of chronic pancreatitis, pancreatic carcinoma or pancreatic surgery. Maldigestion of fat can result in steatorrhoea and weight loss but may not be clinically apparent. Steatorrhoea can be caused by small bowel malabsorption and clinical assessment of steatorrhoea (eg, by stool inspection) is unreliable. ${ }^{175}$ Symptoms of pancreatic exocrine insufficiency usually develop 10-15 years after first symptoms of chronic pancreatitis and when secretion of lipase and other pancreatic enzymes is reduced to $<10 \%$ of normal values. The diagnosis of advanced chronic pancreatitis in patients presenting with diarrhoea is often suggested by the clinical history and confirmed with imaging. A number of recent guidelines outline the current strategies for diagnosing chronic pancreatitis. ${ }^{176-179}$ There are international variations in practice depending on the availability of various modalities such as endoscopic ultrasound (EUS) and direct pancreatic function tests. However, there is a broad agreement that invasive direct pancreatic function testing has become largely redundant in routine clinical practice, replaced by indirect pancreatic function testing and imaging. It is also recognised that there is currently no ideal test to diagnose chronic pancreatitis in early/mild disease.

\subsubsection{Faecal elastase}

Three-day faecal fat estimation is laborious, unpleasant and is no longer performed in UK laboratories. Stool chymotrypsin assays have been replaced by faecal elastase because of its greater stability and the improved sensitivity of the test.

Human elastase- 1 is an anionic protease belonging to the family of serine proteases along with other digestive enzymes such as chymotrypsin and trypsin. Its ability to degrade elastin is unique, but it is also stable in stool for up to a week at room temperature reaching concentrations 5-6 times higher than duodenal juice. This stool test has emerged as a preferred test of pancreatic function and has largely replaced invasive and indirect pancreatic function tests in many centres. Normal values are $200-500 \mu \mathrm{g} / \mathrm{g}$, with $100-200 \mu \mathrm{g} / \mathrm{g}$ in mild to moderate insufficiency and $<100 \mu \mathrm{g} / \mathrm{g}$ in severe insufficiency. The test is unaffected by simultaneous enzyme therapy or diet and requires only a single $100 \mathrm{mg}$ stool sample. Faecal elastase- 1 has been well characterised as a sensitive biomarker for moderate to severe pancreatic insufficiency with sensitivities of $73-100 \%$ and specificities of $80-100 \% .{ }^{180}{ }^{181}$ However, faecal elastase is not useful in cases of mild pancreatic insufficiency with sensitivities of $<60 \%$ and is not able to reliably distinguish pancreatic from non-pancreatic malabsorption. A comparison of faecal elastase with other non-invasive tests suggests superior sensitivity to the para-amino benzoic acid test ${ }^{182}$ and the pancreolauryl test. ${ }^{183}$ The test should be used with care in patients with liquid stool and this can lead to false positive results (ie, low levels of faecal elastase due to dilution).

\subsection{Pancreatic imaging}

The progressive inflammatory change that occurs in chronic pancreatitis leads to significant changes in pancreatic structure. Focal segmental or diffuse destruction of the parenchyma occurs in the early stages while structuring, dilation of the duct and calcification tend to occur late in the disease. There has been debate around the significance of the sometimes subtle changes found at EUS or endoscopic retrograde pancreatography (ERP) in the diagnosis of early chronic pancreatitis, particularly in patients presenting with abdominal pain. For more advanced pancreatic disease with significant morphological change, CT scanning has become the initial imaging of choice given the poor sensitivity of USS in this situation (approximately 60\%) ${ }^{184} 185$ and in assessing for pancreatic malignancy. Its sensitivity in detecting early pancreatic changes is unknown, but it is significantly less than EUS or magnetic resonance cholangiopancreatography (MRCP)/ ERP. Newer non-invasive methods for diagnosing pancreatic cancer are beginning to emerge. ${ }^{186}$

MRCP with secretin (MRCP-S) appears to significantly increase the diagnostic yield in patients with asymptomatic hyperamylasaemia ${ }^{187}$ and showed equivalent sensitivity to endoscopic retrograde cholangio-pancreatography (ERCP) in determining ductal changes in patients with idiopathic recurrent pancreatitis. ${ }^{188}$ The technique has been proposed as an alternative to direct pancreatic function tests, but issues of standardisation and interpretation remain to be resolved. ${ }^{189190}$ Quantitative MRCP after secretin has also been correlated with faecal elastase and pancreolauryl testing ${ }^{191} 192$ and has been shown to correlate with histology. ${ }^{193}$ However, it is a specialised procedure and not recommended for initial diagnostic investigation for chronic pancreatitis.

EUS is able to detect mild parenchymal and ductal abnormalities not seen on CT. Comparison with ERCP in seven studies revealed sensitivity of $68-100 \%$ with specificity of $78-97 \%{ }^{194}$ based on standard scoring of nine criteria. A study of 83 patients showed EUS to have an equivalent diagnostic accuracy for early and late stage chronic pancreatitis when compared with secretin direct 
pancreatic function tests. ${ }^{195} 196$ Two studies comparing MRCP-S and EUS with ERCP have suggested a slightly higher sensitivity of EUS for changes suggestive of chronic pancreatitis, ${ }^{197} 198$ but it remains questionable whether some of the subtle findings at EUS have clinical relevance. Based on these and other studies, both EUS and MRCP-S would seem effective diagnostic tests for chronic pancreatitis, which are best used in a complementary fashion.

Local availability will inevitably dictate the choice of test, and both modalities would seem to be useful in assessing patients with early/mild forms of chronic pancreatitis, particularly if other approaches such as CT or pancreatic function tests are negative or equivocal. MRI with MRCP protocol is more sensitive and accurate in the detection of chronic pancreatitis, as also recommended by the American Pancreatic Association. ${ }^{176}$

\subsection{Invasive pancreatic function testing}

Invasive tests of pancreatic function involving direct measurement of pancreatic secretions (catheter aspiration of secretions after secretin, cholecystokinin stimulation or a standard test meal-the 'Lundh test') have historically been regarded as the gold standard for determining exocrine pancreatic function. 199200 These tests are reported to correlate findings at ERCP, ${ }^{201202}$ but some of these data are now more than three decades old with no recent re-evaluations of these earlier studies. Moreover, these tests are difficult to perform and compare as there is little standardisation. Although American guidelines continue to recommend direct pancreatic function tests as part of the diagnostic investigation for chronic pancreatitis, ${ }^{176}$ there is no place in UK and European practice for these invasive tests.

\subsection{Non-invasive pancreatic function testing}

These tests assess pancreatic enzymes or the consequences of maldigestion by measurement of stool, serum, urine or breath. The tests include fat, elastase and chymotrypsin measurement in stool, urine tests requiring detection of markers in urine or breath released from the gut and absorbed following digestion of synthetic substrate by pancreatic enzymes. All of these tests require significant loss of pancreatic function before becoming positive, with poor sensitivity and specificity in mild/early pancreatic disease.

\subsubsection{Urine and breath tests}

The two tests (NBTP and pancreolauryl) previously in use are now of largely historical interest as neither, to our knowledge, is in use in the UK due to their poor sensitivity and specificity. ${ }^{203}$

Equally, while European guidelines have recommended the use of the C13 mixed triglyceride breath test as an alternative to faecal elastase testing, it is not widely available with poor sensitivity in mild/moderate disease ${ }^{204}$ and therefore not recommended.

\subsection{Small bowel bacterial overgrowth (SBBO) 6.5.1 Introduction}

The small bowel normally has little bacterial colonisation with concentrations in the proximal jejunum of less than $10^{4}$ colony forming units $(\mathrm{cfu}) / \mathrm{mL}$ in the normal healthy state compared with $10^{9}-10^{12}$ in the colon.

Few data exist on the prevalence of small bowel bacterial overgrowth (SBBO) in patients presenting with diarrhoea and/or malabsorption. However, patients with anatomical or functional reasons for abnormal orocaecal transit have been reported to be at increased risk. Such predisposing factors include diabetes, scleroderma, intestinal pseudo-obstruction, prior surgery (eg, terminal ileal resection), diverticulosis or strictures of the small bowel. $^{205}$ Gastric surgery, in particular that involving a blind loop, is associated with a high prevalence of SBBO: up to $50 \%$ of patients with gastrojejunostomy and vagotomy compared with $5 \%$ of those with vagotomy and pyloroplasty, ${ }^{206}$ although the clinical significance of this finding is unclear. ${ }^{207}$ Authors have also reported a high prevalence of SBBO in situations associated with achlorhydria such as old age or medical therapy with proton pump inhibitors. ${ }^{208}$ However, the clinical relevance of SBBO in this context has not been demonstrated. ${ }^{209} 210$

Part of the difficulty in establishing a confident diagnosis of SBBO is the lack of a standardised investigative tool. Culture of a small bowel aspirate is the most direct method of investigation of bacterial overgrowth and has been considered to be the gold standard for diagnosis of this condition by some authors. ${ }^{211}$ The presence of $>10^{6} \mathrm{cfu} / \mathrm{mL}$ in either aerobic or anaerobic conditions is conventionally regarded as the criterion for a positive culture, although lower thresholds have been proposed. However, bacterial overgrowth, particularly due to coliforms and enterococci, may occur in apparently healthy individuals with no evidence of malabsorption, ${ }^{210}$ so the clinical relevance of such a positive result may be difficult to determine. Although malabsorptive syndromes have primarily been associated with anaerobic organisms, isolation and categorisation of bacterial anaerobes are not routinely performed in many laboratories. Furthermore, the lack of standardisation of bacterial counts, the possibility of sampling errors and the need for intubation make a simpler, less invasive investigation desirable.

\subsubsection{Breath tests}

Breath tests have been an attractive alternative to culture of small bowel aspirates for many years. However, the sensitivity and specificity of these tests are in general poor.

Hydrogen breath testing is based on the ability of some bacteria to ferment carbohydrates with an end product of hydrogen, which is not produced by mammalian cells. It was originally proposed that breath testing after a carbohydrate load resulted in a double peak due to metabolism by small bowel bacteria, followed by a more prolonged peak due to metabolism by colonic bacteria. ${ }^{212}$ The reproducibility of this double peak pattern has been challenged ${ }^{213214}$ as the appearance of the initial peak is more likely to be due to fermentation by oropharyngeal flora or variation in orocaecal transit times and fermentation of carbohydrate in the caecum. ${ }^{215}$ In addition, a false negative result may occur in the $3-25 \%$ of individuals whose bacterial flora are not hydrogen producers. ${ }^{216}$ This may in part be due to variations in the particular species of bacteria involved in small bowel colonisation as, for example, none of Staphylococcus aureus, Enterococci spp, Serratia or Pseudomonas spp produce hydrogen.

Because of these problems, it is unsurprising that several studies have now shown the sensitivity and specificity of hydrogen breath tests to be low. ${ }^{211217}$ Recently, Erdogan et al $^{217}$ found that glucose breath testing had a sensitivity less than 50\% compared with duodenal aspirate, regardless of whether or not methane measurement was added to hydrogen. Both the positive and negative predictive values for breath testing were less than $70 \%$. Corazza and colleagues $^{211}$ found sensitivities of $62 \%$ and $68 \%$ for glucose and lactulose-hydrogen breath tests, respectively. These studies support the poor results with the $10 \mathrm{~g}$ lactulose test reported by Riordan and colleagues, ${ }^{218}$ with a sensitivity of only $17 \%$ and a specificity of $70 \%$. The authors used scintigraphy to 
Recommendations

- We recommend faecal elastase testing as the preferred noninvasive test for pancreatic function (Grade of evidence level 1 , Strength of recommendation strong).

- MRI (with MRCP protocol) is the recommended investigation of choice for diagnosing chronic pancreatitis. If unavailable, CT would be the next modality of choice (Grade of evidence level 2, Strength of recommendation strong).

- In cases where pancreatic insufficiency is strongly suspected but initial screening tests are negative, we suggest further imaging with either EUS or MRCP with secretin (if available) (Grade of evidence level 3, Strength of recommendation weak).

- We suggest culture of small bowel aspirates as it is the most sensitive test for small bowel bacterial overgrowth (SBBO), but methods are poorly standardised and positive results may not reflect clinically significant SBBO (Grade of evidence level 2 , Strength of recommendation weak).

- In the absence of an optimal test to confirm the presence of bacterial overgrowth and in those with a high test probability of SBBO, we recommend an empirical trial of antibiotics; the value of this approach has not been subject to definitive study (Grade of evidence level 3, Strength of recommendation strong).

aid interpretation of the breath test, and this increased sensitivity to $39 \%$ and specificity to $100 \% .{ }^{219}{ }^{220}$

This illustrates a key underlying flaw of breath testing in isolation: that transit time is assumed rather than measured. The result of this assumption is that fast orocaecal transit can confound interpretation of an early hydrogen peak, a circumstance that is more likely after intestinal resection. This issue has been demonstrated in high-quality physiological and clinical studies with both lactulose and glucose. ${ }^{214} 219220$

The methodological problems and performance of hydrogen breath testing mean that it may add little when SBBO is suspected. A positive test will reinforce the clinician's prior view that the cause may be SBBO; a negative test will not exclude the diagnosis as a possibility. It is not clear that such an intermediate investigative step is necessary or of value. It would be reasonable to test for SBBO in patients with diarrhoea and a low to moderate pretest probability of SBBO. However, where the pretest probability is high-for example, in those with anatomical abnormalities such as dilation, diverticulosis, prior small bowel surgery or pseudo-obstruction-the most practical approach would be to proceed straight to an empirical antibiotic trial.

Strengthening facilities for microbiological analysis of gut flora after endoscopic sampling is likely to be a more productive focus for service development. Culture of unwashed mucosal biopsies may facilitate collection of microbiological samples rather than by using jejunal aspirates. ${ }^{221}$ In its absence, most units will remain reliant on assessing the effect of an empirical trial of antibiotics. ${ }^{222}$

\section{SURGICAL/STRUCTURAL CAUSES OF DIARRHOEA}

There is significant overlap of the type of patient referred to a surgeon rather than a gastroenterologist and many of the assessment and investigation recommendations are relevant. Often patients are directed towards surgeons through the urgent pathway where there is a need to exclude organic disease. In respect of these patients, investigations should follow the same assessment and investigation pathway as detailed above and in the NICE guidance CG131 (https://www.nice.org.uk/guidance/ cg131). There is a tendency-once organic disease, particularly neoplasia and IBD, have been excluded-for the surgeon to either reassure the patient and discharge or refer on to a gastroenterologist. This may be appropriate but it is prudent for the surgeon to bear in mind other potential organic aetiologies. For instance, unrecognised cases of coeliac disease are sometimes seen in colorectal clinics. Patients present with vague GI symptoms, iron deficiency anaemia or chronic diarrhoea and are often falsely diagnosed as diarrhoea-predominant IBS. ${ }^{223}$

\subsection{Faecal incontinence}

Some groups of patients are directed towards surgeons because diarrhoea is not the primary patient-perceived symptom. A typical example is faecal incontinence. Such patients may well have a compromised sphincter complex but fail to maintain continence because of the underlying diarrhoea. Although an important risk factor for incontinence along with age, obstetric trauma, pelvic surgery, obesity and other medical conditions such as diabetes and stroke, it may not be recognised and investigated appropriately. Again, NICE gives guidance as to appropriate management with baseline assessment aimed at addressing underlying conditions such as potentially treatable causes of diarrhoea (https://www.nice.org.uk/guidance/cg49). An associated group who may complain of both faecal incontinence and diarrhoea are those with severe faecal loading where the diarrhoea is essentially overflow. Typical patients tend to be those with cognitive or behavioural issues, have learning difficulties, or those with neurological or spinal disease. A focused baseline assessment should therefore include an anorectal examination which will identify impaction and, for instance, rectal prolapse. Plain radiography or radio-opaque marker studies have been used but there is poor correlation between the two. ${ }^{224}$ Specialist investigations include anorectal manometry and endoanal ultrasound, but there are no accepted standards for performing these specialist tests. The evidence behind their use is limited to the fact that they are better than simple clinical assessment in deciding surgical management. Therefore, they should only be instigated once conservative measures for faecal incontinence have been exhausted and surgical intervention is contemplated.

\subsection{Post-surgical diarrhoea}

There is a third group of 'surgical' patients with diarrhoea. These are the patients who have developed diarrhoea as a result of surgical intervention. The underlying aetiology of the diarrhoea may be attributed to pyloric dysfunction, bile salt malabsorption, bacterial overgrowth or gut bypass reducing absorptive capacity.

Upper GI surgery may result in damage or division of the vagus nerve accompanied in part by reduction in capacity of the stomach in the case of gastric resection. Rapid gastric emptying may result in osmotic diarrhoea and associated symptoms of 'dumping' syndrome. Surgery can also provide an ideal environment for bacterial colonisation and overgrowth leading to chronic diarrhoea. GI tract surgeries that create a blind loop (eg, a Billroth II procedure or a Roux-en-Y anastomosis) may predispose to stasis and overgrowth due to abnormal motility and ineffective clearance of retained food and secretions. ${ }^{225}$ In addition, those who have undergone jejunoileal bypass, an end-toside enteroenteric anastomosis or the creation of a Koch distal ileal pouch are at risk. In the same way, small bowel strictures may lead to bacterial stasis and overgrowth. These may occur 


\section{Recommendations}

- Faecal impaction with overflow diarrhoea should be considered especially in the elderly. We recommend clinical judgement rather than marker studies to confirm this (Grade of evidence level 4, Strength of recommendation strong).

- For patients with persistent faecal incontinence we recommend anorectal manometry and endoanal ultrasonography once conservative measures have been exhausted (Grade of evidence level 3, Strength of recommendation strong).

- If a fistula is suspected, cross-sectional imaging with contrast is recommended (Grade of evidence level 3, Strength of recommendation strong).

after surgery but can also be seen after radiotherapy, in Crohn's disease and secondary to some medications. ${ }^{226}$

Small and large bowel resection clearly reduces absorptive capacity. At the extreme, this results in short bowel syndrome characterised clinically by chronic diarrhoea, dehydration, electrolyte abnormalities and malnutrition. The severity and management depend on the site and extent of the intestinal resection, whether there is disease in the residual bowel and the degree of adaptation of the remaining bowel. However, even a simple limited right hemicolectomy may result in diarrhoea as a result of loss of the ileocaecal valve and resorptive capacity of the right colon. This increases the risk of developing bacterial overgrowth because of retrograde movement of bacteria from the colon into the small intestine. This has been clearly demonstrated in one trial of patients with Crohn's disease which showed that resection of the ileocaecal valve almost doubled the prevalence of bacterial overgrowth. ${ }^{227}$

\subsection{Fistulae}

The common theme in many of these surgical conditions is structural change. However, structural change may not necessarily be surgically induced. Disease processes can also predispose to gut bypass, bile acid diarrhoea and bacterial overgrowth. Fistula formation, such as commonly seen in Crohn's disease, is one example of gut bypass. Cholecystoenteric fistulae are a potential cause of bile acid diarrhoea, particularly if the fistula is to the colon. ${ }^{228}$ Small bowel diverticula which occur in approximately $1-6 \%$ of the population may harbour bacteria and result in bacterial overgrowth. ${ }^{229}$

\subsection{Investigations}

In terms of investigation, clearly surgically-induced structural change may be obvious from the history. A digital rectal examination is essential. It allows clinical assessment of the sphincter complex and will exclude faecal impaction as well as low anorectal lesions. Further investigations, if required, to examine for structural change are mainly radiological; CT scanning and contrast studies will identify the structural changes.

\section{RARE CAUSES}

\subsection{Neuroendocrine tumours}

Hormone secreting tumours arising from gastroenteropancreatic tissue are rare causes of diarrhoea. The prevalence of functional pancreatic endocrine tumours is approximately 10 per million population, the incidence ranging from $1 / 10^{6}$ cases per year in the case of gastrinomas to fewer than $1 / 10^{7}$ cases per year for vasoactive intestinal peptide (VIP)omas and glucagonomas. ${ }^{230}$ Even this incidence value is likely to be an overestimate. Diarrhoea occurs as part of a symptom complex varying according to the tumour type (eg, 100\% cases in VIPoma, approximately $65 \%$ in gastrinoma). Although diarrhoea has been reported at a prevalence of $15 \%$ in glucagonoma, this again is probably an overestimate. A wide variety of other symptoms may accompany hypersecretion of these hormones and detailed discussion is available elsewhere. ${ }^{231}$

Confirmation of the diagnosis in each case requires demonstration of an elevated serum hormone concentration. A VIP secreting tumour may be suspected in the context of large volumes of secretory diarrhoea ( $>1$ litre/day), dehydration and hypokalaemia. Normal values for circulating VIP are less than $170 \mathrm{pg} / \mathrm{mL}$, while mean VIP serum concentrations in patients with functioning tumours range from 675 to $965 \mathrm{pg} / \mathrm{mL} .{ }^{232} \mathrm{As}$ serum levels fluctuate, the assay should be performed during an episode of diarrhoea. Similarly, serum gastrin levels in patients with gastrinomas are considerably higher than the normal range of $150 \mathrm{pg} / \mathrm{mL}$, with average values of approximately $1000 \mathrm{pg} /$ $\mathrm{mL}$. However, comparable values can be found in patients with pernicious anaemia, other types of atrophic gastritis or potent acid suppressant therapy. ${ }^{233}$ Raised levels, although not to the same degree, also occur in other conditions such as diabetes mellitus, renal insufficiency and rheumatoid arthritis.

Diarrhoea is often a prominent feature (occuring in up to $50 \%$ of cases $^{234}$ ) in carcinoid syndrome, which accounts for $20 \%$ of midgut neuroendocrine tumours. ${ }^{235}$ This almost always occurs in the context of hepatic metastases, even if the primary site remains undefined. The clinical diagnosis of 'malignant disease' is usually evident. A raised 24-hour urinary 5-hydroxyindoleacetic acid (above the local reference laboratory value) has a high sensitivity and specificity $(88 \%)^{236}$ for the condition and correlates with tumour bulk, bioactive product secretion and, frequently, with the severity of symptoms. ${ }^{237}$ However, this is subject to patients adhering to dietary restrictions and often a 3-day dietary intake should accompany the sample so the laboratory can assess for any dietary interference (eg, serotonin-rich food such as bananas). ${ }^{235}$

\subsection{Factitious diarrhoea}

Factitious diarrhoea caused by laxative abuse or the spurious adding of water or urine to stool specimens has been recognised as a relatively common cause of reported chronic diarrhoeal symptoms in Western populations. The likelihood of this diagnosis increases as more numerous and repeated investigations reveal negative results. Thus, although only $4 \%$ of patients visiting district gastroenterology clinics had factitious diarrhoea, this value increased to $20 \%$ of those evaluated at tertiary referral centres, which made it the most common cause of diarrhoea of previously undetermined origin. ${ }^{15}$ Similarly, a survey of patients who had undergone extensive evaluation revealed 33\% who were found to be taking laxatives or diuretics; $22 \%$ had undiagnosed colitis (ulcerative or microscopic colitis), $7 \%$ had faecal incontinence and $7 \%$ had other organic disorders. ${ }^{26}$

Repeated analysis of stool and urine is wise, as patients may ingest laxatives intermittently. Alkalinisation assays, although simple to use (phenolphthalein, some anthraquinones and rhubarb turn the stool red, bisacodyl turns it purple-blue), are not of sufficient sensitivity and should be abandoned. A screen for 'laxative abuse' should include the detection of anthraquinones, bisacodyl and phenolphthalein in urine and magnesium and phosphate in stool, and should be carried out in a specialist 
Recommendations

- Hormone secreting tumours in the absence of other findings are a rare cause of diarrhoea and we recommend testing only if all other causes have been sought (Grade of evidence level 4 , Strength of recommendation strong).

- Factitious diarrhoea can be difficult to confirm but, if suspected, we suggest a stool screen for laxative abuse (Grade of evidence level 5, Strength of recommendation weak).

laboratory. Laboratories in the UK performing these tests should participate in the UK External Quality Assessment scheme for the detection of laxatives.

\section{SUMMARY AND CONCLUSIONS}

Establishing a clear definition of diarrhoea based on history alone can prove difficult, and this tends to lead to overinvestigation of functional diarrhoea including IBS. Serological testing for coeliac disease, the most common small bowel enteropathy in Caucasian populations, should be performed early in the course of investigations. The initial assessment should direct the clinician to determine whether further investigation is necessary and, if so, whether the focus should be on colonic, small bowel or pancreatic disease. This analysis could reasonably be performed in the primary care setting.

Most chronic diarrhoea is due to colonic abnormalities. Colonic investigations should be age stratified, in keeping with the risk of neoplasia and inflammation, and the high prevalence of bile acid diarrhoea estimated to be similar to coealic disease should be recognised and investigated. After appropriate normal investigations, those with persistent diarrhoea are likely to be self-limiting 'idiopathic' diarrhoea or undiagnosed factitious diarrhoea. ${ }^{26} 238$ Since the overall prognosis appears to be good in this group, including those that self-report and self-remedy, ${ }^{239}$ further investigation in this group is not warranted and symptomatic treatment could be instituted.

\section{Author affiliations}

${ }^{1}$ University Hospital Coventry, Coventry, UK

${ }^{2}$ Department of Applied Biological Sciences, University of Coventry, Coventry, UK

${ }^{3}$ Clinical Sciences Research Institute, University of Warwick, Warwick, UK

${ }^{4}$ Sheffield Teaching Hospitals, Sheffield, UK

${ }^{5}$ Norwich Medical School, University of East Anglia, Norwich, UK

${ }^{6}$ University of Zürich, Zürich, Switzerland

${ }^{7}$ Abdominal Centre, St Claraspital, Basel, Switzerland

${ }^{8}$ School of Medicine, Pharmacy \& Health, University of Durham, Durham, UK

${ }^{9}$ Patient Representative, Secretary BAM Support, Coventry, UK

${ }^{10} \mathrm{NIHR}$ Nottingham Biomedical Research Centre (BRC), Nottingham University

Hospitals NHS Trust and the University of Nottingham, Nottingham, UK

${ }^{11}$ Department of Radiology, South Warwickshire Hospitals, Warwick, UK

${ }^{12}$ Department of Clinical Biochemistry, Midlands and NW Bowel Cancer Screening Hub, Rugby, UK

${ }^{13}$ Department of Gastroenterology, Musgrave Park Hospital, Taunton, UK

${ }^{14}$ Division of Digestive Diseases, Imperial College London, London, UK

Contributors All authors formed part of the Guideline Development Group (GDG) and contributed equally to this manuscript.

Funding This research received no specific grant from any funding agency in the public, commercial or not-for-profit sectors.

Competing interests None declared.

Patient consent Not required.

Provenance and peer review Not commissioned; externally peer reviewed.

Open access This is an open access article distributed in accordance with the Creative Commons Attribution Non Commercial (CC BY-NC 4.0) license, which permits others to distribute, remix, adapt, build upon this work non-commercially, and license their derivative works on different terms, provided the original work is properly cited and the use is non-commercial. See: http://creativecommons.org/ licenses/by-nc/4.0/

(C) Article author(s) (or their employer(s) unless otherwise stated in the text of the article) 2018. All rights reserved. No commercial use is permitted unless otherwise expressly granted.

\section{REFERENCES}

1 Brouwers MC, Kho ME, Browman GP, et al. AGREE II: advancing guideline development, reporting and evaluation in health care. Can Med Assoc J 2010;182:E839-E842.

2 Guyatt G, Oxman AD, AkI EA, et al. GRADE guidelines: 1. IntroductionGRADE evidence profiles and summary of findings tables. J Clin Epidemiol 2011;64:383-94.

3 Oxford University. Centre for evidence-based medicine. Oxford: Oxford University, 2009.

4 Lacy BE, Mearin F, Chang L, et al. Bowel disorders. Gastroenterology 2016:150:1393-407.

5 Wenzl HH, Fine KD, Schiller LR, et al. Determinants of decreased fecal consistency in patients with diarrhea. Gastroenterology 1995;108:1729-38.

6 O'Donnell LJ, Virjee J, Heaton KW. Detection of pseudodiarrhoea by simple clinical assessment of intestinal transit rate. BMJ 1990;300:439-40.

7 Madoff RD, Williams JG, Caushaj PF. Fecal incontinence. N Engl J Med 1992;326:1002-7

8 Fine KD, Schiller LR. AGA technical review on the evaluation and management of chronic diarrhea. Gastroenterology 1999;116:1464-86.

9 Stotzer P-O, Abrahamsson H, Bajor A, et al. Are the definitions for chronic diarrhoea adequate? Evaluation of two different definitions in patients with chronic diarrhoea. United Eur Gastroenterol I 2015:3:381-6.

10 Talley NJ, Weaver AL, Zinsmeister AR, et al. Onset and disappearance of gastrointestinal symptoms and functional gastrointestinal disorders. Am J Epidemiol 1992:136:165-77.

11 Manning AP, Thompson WG, Heaton KW, et al. Towards positive diagnosis of the irritable bowel. BMJ 1978;2:653-4.

12 Sood R, Camilleri M, Gracie DJ, et al. Enhancing diagnostic performance of symptom-based criteria for irritable bowel syndrome by additional history and limited diagnostic evaluation. Am J Gastroentero/ 2016;111:1446-54.

13 Canavan C, West J, Card T. The epidemiology of irritable bowel syndrome. Clin Epidemiol 2014;6:71-80

14 Lovell RM, Ford AC. Global prevalence of and risk factors for irritable bowel syndrome: a meta-analysis. Clin Gastroenterol Hepatol 2012;10:712-21.

15 Duncan A, Hill PG. A UK survey of laboratory-based gastrointestinal investigations. Ann Clin Biochem 1998;35(Pt 4):492-503.

16 Jones R. Primary care research and clinical practice: gastroenterology. Postgrad Med J 2008:84:454-8

17 Schiller LR, Pardi DS, Spiller R, et al. Gastro 2013 APDW/WCOG Shanghai Working Party Report: Chronic diarrhea: Definition, classification, diagnosis. J Gastroenterol Hepatol 2014;29:6-25

18 Whitehead WE, Palsson OS, Simrén M. Irritable bowel syndrome: what do the new Rome IV diagnostic guidelines mean for patient management? Expert Rev Gastroenterol Hepatol 2017;11:281-3.

19 Talley NJ, Phillips SF, Melton LJ, et al. Diagnostic value of the Manning criteria in irritable bowel syndrome. Gut 1990;31:77-81.

20 Thompson WG. Gastrointestinal symptoms in the irritable bowel compared with peptic ulcer and inflammatory bowel disease. Gut 1984;25:1089-92.

21 Arrambide KA, Santa Ana CA, Schiller LR, et al. Loss of absorptive capacity for sodium chloride as a cause of diarrhea following partial ileal and right colon resection. Dig Dis Sci 1989;34:193-201.

22 Ros E, Zambon D. Postcholecystectomy symptoms. A prospective study of gall stone patients before and two years after surgery. Gut 1987;28:1500-4.

23 Valdovinos MA, Camilleri M, Zimmerman BR. Chronic diarrhea in diabetes mellitus: mechanisms and an approach to diagnosis and treatment. Mayo Clin Proc 1993:68:691-702.

24 Person J. Alcohol and the small intestine. Scand J Gastroenterol 1991;26:3-15.

25 Jain NK, Rosenberg DB, Ulahannan MJ, et al. Sorbitol intolerance in adults. Am J Gastroenterol 1985;80:678-81.

26 Read NW, Krejs GJ, Read MG, et al. Chronic diarrhea of unknown origin. Gastroenterology 1980;78:264-71.

27 Ackerman Z, Eliakim R, Stalnikowicz R, et al. Role of small bowel biopsy in the endoscopic evaluation of adults with iron deficiency anemia. Am J Gastroenterol 1996:91:2099-102.

28 Goddard AF, McIntyre AS, Scott BB. Guidelines for the management of iron deficiency anaemia. British Society of Gastroenterology. Gut 2000;46(Suppl 3-4):IV1-IV5.

29 American Gastroenterological Association. Medical position statement: celiac sprue. Gastroenterology 2001;120:1522-5. 
30 West J, et al. Seroprevalence, correlates, and characteristics of undetected coeliac disease in England. Gut 2003;52:960-5.

31 Fasano A, Berti I, Gerarduzzi T, et al. Prevalence of celiac disease in at-risk and notat-risk groups in the United States. Arch Intern Med 2003;163:286-92.

32 Green PH, Jabri B. Coeliac disease. Lancet 2003;362:383-91.

33 Catassi C, Kryszak D, Bhatti B, et al. Natural history of celiac disease autoimmunity in a USA cohort followed since 1974. Ann Med 2010;42:530-8.

34 Lohi S, Mustalahti K, Kaukinen K, et al. Increasing prevalence of coeliac disease over time. Aliment Pharmacol Ther 2007;26:1217-25.

35 Rubio-Tapia A, Kyle RA, Kaplan EL, et al. Increased prevalence and mortality in undiagnosed celiac disease. Gastroenterology 2009;137:88-93.

36 Green PHR. The many faces of celiac disease: Clinical presentation of celiac disease in the adult population. Gastroenterology 2005;128(4):S74-S78.

37 Fasano A, Catassi C. Current approaches to diagnosis and treatment of celiac disease: An evolving spectrum. Gastroenterology 2001;120:636-51.

38 Hopper AD, Hadjivassiliou M, Butt $\mathrm{S}$, et al. Adult coeliac disease. BMJ 2007;335:558-62.

39 Ferguson A, Arranz E, O'Mahony S. Clinical and pathological spectrum of coeliac disease--active, silent, latent, potential. Gut 1993:34:150-1.

40 Sanders DS, Carter MJ, Hurlstone DP, et al. Association of adult coeliac disease with irritable bowel syndrome: a case-control study in patients fulfilling ROME II criteria referred to secondary care. Lancet 2001;358:1504-8.

41 Sanders DS, et al. Changing face of adult coeliac disease: experience of a single university hospital in South Yorkshire. Postgrad Med J 2002;78:31-3.

42 Green PHR, Stavropoulos SN, Panagi SG, et al. Characteristics of adult celiac disease in the USA: results of a national survey. Am J Gastroentero/ 2001;96:126-31.

43 Richey R, Howdle P, Shaw E, et al. [Recognition and assessment of celiac disease in children and adults: summary of NICE guideline]. Praxis 2009;98:1233-5.

44 Cranney A, Zarkadas M, Graham ID, et al. The Canadian Celiac Health Survey. Dig Dis Sci 2007:52:1087-95.

45 Lo W, Sano K, Lebwohl B, et al. Changing presentation of adult celiac disease. Dig Dis Sci 2003;48:395-8.

46 Fernández-Bañares F, Esteve M, Salas A, et al. Systematic evaluation of the causes of chronic watery diarrhea with functional characteristics. Am J Gastroenterol 2007:102:2520-8.

47 Hopper AD, Cross SS, Hurlstone DP, et al. Pre-endoscopy serological testing for coeliac disease: evaluation of a clinical decision tool. BMJ 2007:334:729.

48 Hill ID. What are the sensitivity and specificity of serologic tests for celiac disease? Do sensitivity and specificity vary in different populations? Gastroenterology 2005;128(4):S25-S32

49 Tursi A, Giorgetti G, Brandimarte G, et al. Prevalence and clinical presentation of subclinical/silent celiac disease in adults: an analysis on a 12-year observation. Hepatogastroenterology 2001;48:462-4.

50 Collin $\mathrm{P}$, Kaukinen $\mathrm{K}$, Vogelsang $\mathrm{H}$, et al. Antiendomysial and antihuman recombinant tissue transglutaminase antibodies in the diagnosis of coeliac disease: a biopsyproven European multicentre study. Eur J Gastroenterol Hepatol 2005;17:85-91.

51 Rostami K, Kerckhaert J, Tiemessen R, et al. Sensitivity of antiendomysium and antigliadin antibodies in untreated celiac disease: disappointing in clinical practice. Am J Gastroentero/ 1999;94:888-94.

52 Tursi A, Brandimarte G, Giorgetti GM. Prevalence of antitissue transglutaminase antibodies in different degrees of intestinal damage in celiac disease. J Clin Gastroenterol 2003;36:219-21.

53 Harrison E, Li K-K, Petchey M, et al. Selective measurement of anti-tTG antibodies in coeliac disease and IgA deficiency: an alternative pathway. Postgrad Med J 2013;89:4-7.

54 Elfstrand L, Florén CH. Management of chronic diarrhea in HIV-infected patients: current treatment options, challenges and future directions. HIV/AIDS 2010;2:219-24.

55 Isaac-Renton JL. Laboratory diagnosis of giardiasis. Clin Lab Med 1991;11:811-27.

56 Rosenblatt JE, Sloan LM, Schneider SK. Evaluation of an enzyme-linked immunosorbent assay for the detection of Giardia lamblia in stool specimens. Diagn Microbiol Infect Dis 1993;16:337-41.

57 Mank TG, Zaat JOM, Deelder AM, et al. Sensitivity of microscopy versus enzyme immunoassay in the laboratory diagnosis of giardiasis. Eur J Clin Microbiol Infect Dis 1997; 16:615-9.

58 Zaat JOM, Mank TG, Assendelft WJJ. A systematic review on the treatment of giardiasis. Trop Med Int Health 1997;2:63-82.

59 Dhanalakshmi S, Meenachi C, Parija SC. Indirect haemagglutination test in comparison with ELISA for detection of antibodies against invasive amoebiasis. J Clin Diagn Res 2016;10:DC05-8.

60 Cornely OA, Crook DW, Esposito R, et al. Fidaxomicin versus vancomycin for infection with Clostridium difficile in Europe, Canada, and the USA: a double-blind, non-inferiority, randomised controlled trial. Lancet Infect Dis 2012;12:281-9.

61 Louie TJ, Miller MA, Mullane KM, et al. Fidaxomicin versus vancomycin for Clostridium difficile infection. N Eng/ J Med Overseas Ed 2011;364:422-31.

62 Wilcox MH, Gerding DN, Poxton IR, et al. Bezlotoxumab for prevention of recurrent Clostridium difficile infection. N Engl J Med Overseas Ed 2017;376:305-17.
63 Davies KA, Longshaw CM, Davis GL, et al. Underdiagnosis of Clostridium difficile across Europe: the European, multicentre, prospective, biannual, point-prevalence study of Clostridium difficile infection in hospitalised patients with diarrhoea (EUCLID). Lancet Infect Dis 2014:14:1208-19.

64 Wadhwa A, Al Nahhas MF, Dierkhising RA, et al. High risk of post-infectious irritable bowel syndrome in patients with Clostridium difficile infection. Aliment Pharmacol Ther 2016:44:576-82.

65 Schiller LR, Pardi DS, Sellin JH. Chronic diarrhea: diagnosis and management. Clin Gastroenterol Hepatol 2017;15:182-93.

66. Waugh N, Cummins E, Royle $P$, et al. Faecal calprotectin testing for differentiating amongst inflammatory and non-inflammatory bowel diseases: systematic review and economic evaluation. Health Technol Assess 2013;17:xv-xix.

67 Dhaliwal A, Zeino Z, Tomkins C, et al. Utility of faecal calprotectin in inflammatory bowel disease (IBD): what cut-offs should we apply? Frontline Gastroenterol 2015;6:14-19.

68 McFarlane M, Chambers S, Dhaliwal A, et al. Is NICE too optimistic about savings from faecal calprotectin testing? Value Health 2015;18:A623.

69 Tibble JA, Sigthorsson G, Foster R, et al. High prevalence of NSAID enteropathy as shown by a simple faecal test. Gut 1999;45:362-6.

70 Kawashima K, Ishihara S, Yuki T, et al. Fecal calprotectin more accurately predicts endoscopic remission of Crohn's disease than serological biomarkers evaluated using balloon-assisted enteroscopy. Inflamm Bowel Dis 2017;23:2027-34.

71 McFarlane M, Chambers S, Malik A, et al. Clinical outcomes at 12 months and risk of inflammatory bowel disease in patients with an intermediate raised fecal calprotectin: a 'real-world' view. BMJ Open 2016;6:e011041.

72 Karsa LV, Lignini TA, Patnick J, et al. The dimensions of the CRC problem. Best Pract Res Clin Gastroenterol 2010:24:381-96.

73 Mowat C, Digby J, Strachan JA, et al. Faecal haemoglobin and faecal calprotectin as indicators of bowel disease in patients presenting to primary care with bowel symptoms. Gut 2016;65:1463-9.

74 Quyn AJ, Steele RJ, Digby J, et al. Application of NICE guideline NG12 to the initial assessment of patients with lower gastrointestinal symptoms: not FIT for purpose? Ann Clin Biochem 2018;55:69-76

75 Widlak MM, Thomas CL, Thomas MG, et al. Diagnostic accuracy of faecal biomarkers in detecting colorectal cancer and adenoma in symptomatic patients. Aliment Pharmacol Ther 2017;45:354-63.

76 Dalrymple J, Bullock I. Diagnosis and management of irritable bowel syndrome in adults in primary care: summary of NICE guidance. BMJ 2008;336:556-8.

77 Rezaie A, Park SC, Morales W, et al. Assessment of anti-vinculin and anti-cytoletha distending toxin B antibodies in subtypes of irritable bowel syndrome. Dig Dis Sci 2017:62:1480-5.

78 Neugut Al, Garbowski GC, Waye JD, et al. Diagnostic yield of colorectal neoplasia with colonoscopy for abdominal pain, change in bowel habits, and rectal bleeding. Am J Gastroenterol 1993;88:1179-83.

79 Lieberman DA, Weiss DG, Bond JH, et al. Use of colonoscopy to screen asymptomatic adults for colorectal cancer. Veterans Affairs Cooperative Study Group 380. N Engl J Med 2000;343:162-8.

80 Marshall JB, Singh R, Diaz-Arias AA. Chronic, unexplained diarrhea: are biopsies necessary if colonoscopy is normal? Am J Gastroenterol 1995:90:372-6.

81 Shah RJ, Fenoglio-Preiser C, Bleau BL, et al. Usefulness of colonoscopy with biopsy in the evaluation of patients with chronic diarrhea. Am J Gastroenterol 2001:96:1091-5.

82 Shale MJH, Walters JRF, Westaby D. Adequacy of flexible sigmoidoscopy with biopsy for diarrhea in patients under age 50 without features of proximal disease. Gastrointest Endosc 2011;73:757-64.

83 Tanaka M, Mazzoleni G, Riddell RH. Distribution of collagenous colitis: utility of flexible sigmoidoscopy. Gut 1992;33:65-70.

84 Zwas FR, Bonheim NA, Berken CA, et al. Diagnostic yield of routine ileoscopy. Am J Gastroenterol 1995;90:1441-3.

85 Geboes K, Ectors N, D'Haens G, et al. Is ileoscopy with biopsy worthwhile in patients presenting with symptoms of inflammatory bowel disease? Am J Gastroenterol 1998:93:201-6.

86 Winawer SJ, Stewart ET, Zauber AG, et al. A comparison of colonoscopy and doublecontrast barium enema for surveillance after polypectomy. N Engl J Med Overseas Ed 2000:342:1766-72.

87 Arasaradnam RP, Cullis J, Nwokolo C, et al. Bile acid malabsorption and SeHCAT the 'Cinderella' will be going to the Nuclear Medicine Ball!. Nucl Med Commun 2012;33:449-51.

88 Fine KD, Seidel RH, Do K. The prevalence, anatomic distribution, and diagnosis of colonic causes of chronic diarrhea. Gastrointest Endosc 2000;51:318-26.

89 Paulsen SR, Huprich JE, Fletcher JG, et al. CT enterography as a diagnostic tool in evaluating small bowel disorders: review of clinical experience with over 700 cases. Radiographics 2006;26:641-57.

90 Castiglione F, Mainenti PP, De Palma GD, et al. Noninvasive diagnosis of small bowel Crohn's disease: direct comparison of bowel sonography and magnetic resonance enterography. Inflamm Bowel Dis 2013;19:991-8. 
91 Fraquelli M, Colli A, Casazza G, et al. Role of US in detection of Crohn disease: metaanalysis. Radiology 2005;236:95-101.

92 Panés J, Bouzas R, Chaparro M, et al. Systematic review: the use of ultrasonography, computed tomography and magnetic resonance imaging for the diagnosis, assessment of activity and abdominal complications of Crohn's disease. Aliment Pharmacol Ther 2011;34:125-45.

93 Gourtsoyiannis N, Papanikolaou N, Grammatikakis J, et al. MR enteroclysis: technical considerations and clinical applications. Eur Radio/ 2002;12:2651-8.

94 Gourtsoyiannis NC, Grammatikakis J, Papamastorakis G, et al. Imaging of small intestinal Crohn's disease: comparison between MR enteroclysis and conventional enteroclysis. Eur Radiol 2006;16:1915-25.

95 Rajesh A, Maglinte DDT. Multislice CT enteroclysis: technique and clinical applications. Clin Radiol 2006;61:31-9.

96 Negaard A, Paulsen V, Sandvik L, et al. A prospective randomized comparison between two MRI studies of the small bowel in Crohn's disease, the oral contrast method and MR enteroclysis. Eur Radiol 2007;17:2294-301.

97 Negaard A, Sandvik L, Berstad AE, et al. MRI of the small bowel with oral contrast or nasojejunal intubation in Crohn's disease: randomized comparison of patient acceptance. Scand I Gastroenterol 2008;43:44-51.

98 Horsthuis K, Bipat S, Bennink RJ, et al. Inflammatory bowel disease diagnosed with US, MR, scintigraphy, and CT: meta-analysis of prospective studies. Radiology 2008;247:64-79.

99 Horsthuis K, Stokkers PCF, Stoker J. Detection of inflammatory bowel disease: diagnostic performance of cross-sectional imaging modalities. Abdom Imaging 2008;33:407-16

100 Ryan ER, Heaslip ISE. Magnetic resonance enteroclysis compared with conventional enteroclysis and computed tomography enteroclysis: a critically appraised topic. Abdom Imaging 2008;33:34-7.

101 Sinha R, Murphy P, Sanders S, et al. Diagnostic accuracy of high-resolution MR enterography in Crohn's disease: comparison with surgical and pathological specimen. Clin Radiol 2013;68:917-27.

102 Oussalah A, Laurent V, Bruot O, et al. Diffusion-weighted magnetic resonance without bowel preparation for detecting colonic inflammation in inflammatory bowel disease. Gut 2010;59:1056-65.

103 Sinha R, Rajiah P, Ramachandran I, et al. Diffusion-weighted MR imaging of the gastrointestinal tract: technique, indications, and imaging findings. Radiographics 2013:33:655-76.

104 Desmond AN, O'Regan K, Curran C, et al. Crohn's disease: factors associated with exposure to high levels of diagnostic radiation. Gut 2008;57:1524-9.

105 Masselli G, Di Tola M, Casciani E, et al. Diagnosis of small-bowel diseases: prospective comparison of multi--detector row CT enterography with MR enterography. Radiology 2016;279:420-31.

106 Pennazio M, Spada C, Eliakim R, et al. Small-bowel capsule endoscopy and device-assisted enteroscopy for diagnosis and treatment of small-bowel disorders: European Society of Gastrointestinal Endoscopy (ESGE) clinical guideline. Endoscopy 2015;47:352-86.

107 Landi B, Tkoub M, Gaudric M, et al. Diagnostic yield of push-type enteroscopy in relation to indication. Gut 1998:42:421-5.

108 Sidhu R, Sanders DS, Morris AJ, et al. Guidelines on small bowel enteroscopy and capsule endoscopy in adults. Gut 2008:57:125-36.

109 Smith MJ, Cherian P, Raju GS, et al. Bile acid malabsorption in persistent diarrhoea. $J$ $R$ Coll Physicians Lond 2000;34:448-51.

110 Bannaga A, Kelman L, O'Connor M, et al. How bad is bile acid diarrhoea: an online survey of patient-reported symptoms and outcomes. BMJ Open Gastroenterol 2017:4:e000116

111 Walters JR. Bile acid diarrhoea and FGF19: new views on diagnosis, pathogenesis and therapy. Nat Rev Gastroenterol Hepatol 2014;11:426-34.

112 Walters JRF. Defining primary bile acid diarrhea: making the diagnosis and recognizing the disorder. Expert Rev Gastroenterol Hepatol 2010;4:561-7.

113 Merrick MV, Eastwood MA, Anderson JR, et al. Enterohepatic circulation in man of a gamma-emitting bile-acid conjugate, 23-selena-25-homotaurocholic acid (SeHCAT). J Nucl Med 1982;23:126-30

114 Merrick MV, Eastwood MA, Ford MJ. Is bile acid malabsorption underdiagnosed? An evaluation of accuracy of diagnosis by measurement of SeHCAT retention. BMJ 1985:290:665-8.

115 Wedlake L, A'HERN R, Russell D, et al. Systematic review: the prevalence of idiopathic bile acid malabsorption as diagnosed by SeHCAT scanning in patients with diarrhoea-predominant irritable bowel syndrome. Aliment Pharmacol Ther 2009:30:707-17.

116 Ross S, D'Mello M, Anand SS, et al. Effect of bile acid sequestrants on the risk of cardiovascular events: a mendelian randomization analysis. Circ Cardiovasc Genet 2015;8:618-27.

117 Sauter GH, Münzing W, von Ritter C, et al. Bile acid malabsorption as a cause of chronic diarrhea: diagnostic value of 7alpha-hydroxy-4-cholesten-3-one in serum. Dig Dis Sci 1999;44:14-19.

118 Camilleri M. Advances in understanding of bile acid diarrhea. Expert Rev Gastroenterol Hepatol 2014;8:49-61.
119 Camilleri M, Busciglio I, Acosta A, et al. Effect of increased bile acid synthesis or fecal excretion in irritable bowel syndrome-diarrhea. Am I Gastroenterol 2014;109:1621-30.

120 Covington J, Westenbrink E, Ouaret N, et al. Application of a novel tool for diagnosing bile acid diarrhoea. Sensors 2013;13:11899-912.

121 Camilleri M. Bile acid diarrhea: prevalence, pathogenesis, and therapy. Gut Liver 2015;9:332-9.

122 Valentin N, Camilleri M, Altayar 0, et al. Biomarkers for bile acid diarrhoea in functional bowel disorder with diarrhoea: a systematic review and meta-analysis. Gut 2016:65:1951-9.

123 Kurien M, Evans KE, Leeds JS, et al. Bile acid malabsorption: an underinvestigated differential diagnosis in patients presenting with diarrhea predominant irritable bowel syndrome type symptoms. Scand I Gastroenterol 2011;46(7-8):818-22

124 Bajor A, Törnblom H, Rudling M, et al. Increased colonic bile acid exposure: a relevant factor for symptoms and treatment in IBS. Gut 2015;64:84-92.

125 Nyhlin H, Merrick MV, Eastwood MA. Bile acid malabsorption in Crohn's disease and indications for its assessment using SeHCAT. Gut 1994;35:90-3.

126 Sciarretta G, Furno A, Mazzoni M, et al. Post-cholecystectomy diarrhea: evidence of bile acid malabsorption assessed by SeHCAT test. Am I Gastroenterol 1992:87:1852-4.

127 Fernández-Bañares F, Esteve M, Salas A, et al. Bile acid malabsorption in microscopic colitis and in previously unexplained functional chronic diarrhea. Dig Dis Sci 2001:46:2231-8

128 Phillips F, Muls ACG, Lalji A, et al. Are bile acid malabsorption and bile acid diarrhoea important causes of loose stool complicating cancer therapy? Colorectal Dis 2015;17:730-4

129 Riemsma R, Al M, Corro Ramos I, et al. SeHCAT [tauroselcholic (selenium-75) acid] for the investigation of bile acid malabsorption and measurement of bile acid pool loss: a systematic review and cost-effectiveness analysis. Health Technol Assess 2013:17:1-236

130 Kurien M, Thurgar E, Davies A, et al. Challenging current views on bile acid diarrhoea and malabsorption. Frontline Gastroenterol 2017:flgastro-2017-100808.

131 Turner JM, Pattni SS, Appleby RN, et al. A positive SeHCAT test results in fewer subsequent investigations in patients with chronic diarrhoea. Frontline Gastroenterol 2017:8:279-83

132 Orekoya $\mathrm{O}$, McLaughlin J, Leitao E, et al. Quantifying bile acid malabsorption helps predict response and tailor sequestrant therapy. Clin Med 2015;15:252-7.

133 Münch A, Aust D, Bohr J, et al. Microscopic colitis: current status, present and future challenges: statements of the European Microscopic Colitis Group. J Crohns Colitis 2012;6:932-45

134 Bogomoletz WV, Adnet JJ, Birembaut P, et al. Collagenous colitis: an unrecognised entity. Gut 1980;21:164-8.

135 Kingham JG, Levison DA, Ball JA, et al. Microscopic colitis: a cause of chronic watery diarrhoea. BMJ 1982;285:1601-4.

136 Langner C, Aust D, Ensari A, et al. Histology of microscopic colitis-review with a practical approach for pathologists. Histopathology 2015;66:613-26.

137 Pardi DS, Kelly CP. Microscopic colitis. Gastroenterology 2011:140:1155-65.

138 Rasmussen MA, Munck LK. Systematic review: are lymphocytic colitis and collagenous colitis two subtypes of the same disease - microscopic colitis? Aliment Pharmacol Ther 2012;36:79-90.

139 Tong J, Zheng Q, Zhang C, et al. Incidence, prevalence, and temporal trends of microscopic colitis: a systematic review and meta-analysis. Am J Gastroenterol 2015;110:265-76. quiz 77

140 Guagnozzi D, Arias Á, Lucendo AJ. Systematic review with meta-analysis: diagnostic overlap of microscopic colitis and functional bowel disorders. Aliment Pharmacol Ther 2016:43:851-62.

141 Kamp EJCA, Kane JS, Ford AC. Irritable bowel syndrome and microscopic colitis: a systematic review and meta-analysis. Clin Gastroenterol Hepatol 2016;14:659-68. e1; quiz e54-5.

142 Nguyen GC, Smalley WE, Vege SS, et al. American Gastroenterological Association Institute guideline on the medical management of microscopic colitis. Gastroenterology 2016;150:242-6. quiz e17-8.

143 Pardi DS, Tremaine WJ, Carrasco-Labra A. American Gastroenterological Association Institute technical review on the medical management of microscopic colitis. Gastroenterology 2016;150:247-74.

144 von Arnim U, Wex T, Ganzert C, et al. Fecal calprotectin: a marker for clinica differentiation of microscopic colitis and irritable bowel syndrome. Clin Exp Gastroenterol 2016:9:97-103.

145 Cotter TG, Binder M, Harper EP, et al. Optimization of a scoring system to predict microscopic colitis in a cohort of patients with chronic diarrhea. J Clin Gastroenterol 2017:51:228-34.

146 Miehlke S, Madisch A, Bethke B, et al. Oral budesonide for maintenance treatment of collagenous colitis: a randomized, double-blind, placebo-controlled trial. Gastroenterology 2008;135:1510-6.

147 Münch A, Langner C. Microscopic colitis: clinical and pathologic perspectives. Clin Gastroenterol Hepatol 2015;13:228-36. 
148 Gibson PR, Shepherd SJ. Evidence-based dietary management of functional gastrointestinal symptoms: The FODMAP approach. J Gastroenterol Hepatol 2010;25:252-8.

149 Major G, Pritchard S, Murray K, et al. Colon hypersensitivity to distension, rather than excessive gas production, produces carbohydrate-related symptoms in individuals with irritable bowel syndrome. Gastroenterology 2017;152:124-33.

150 Shepherd SJ, Parker FC, Muir JG, et al. Dietary triggers of abdominal symptoms in patients with irritable bowel syndrome: randomized placebo-controlled evidence. Clin Gastroenterol Hepatol 2008;6:765-71.

151 Yang J, Deng Y, Chu H, et al. Prevalence and presentation of lactose intolerance and effects on dairy product intake in healthy subjects and patients with irritable bowel syndrome. Clin Gastroenterol Hepatol 2013;11:262-8.

152 Zhu Y, Zheng X, Cong Y, et al. Bloating and distention in irritable bowel syndrome: the role of gas production and visceral sensation after lactose ingestion in a population with lactase deficiency. Am J Gastroentero/ 2013;108:1516-25.

153 Jones HF, Butler RN, Brooks DA. Intestinal fructose transport and malabsorption in humans. Am J Physiol Gastrointest Liver Physiol 2011;300:G202-G206.

154 De Giorgio R, Volta U, Gibson PR, et al. Gluten and FODMAPs in IBS: facts or fiction? Gut 2016;65:169-78.

155 Biesiekierski JR, Peters SL, Newnham ED, et al. No effects of gluten in patients with self-reported non-celiac gluten sensitivity after dietary reduction of fermentable, poorly absorbed, short-chain carbohydrates. Gastroenterology 2013;145:320-8.

156 Deng Y, Misselwitz B, Dai N, et al. Lactose intolerance in adults: biological mechanism and dietary management. Nutrients 2015;7:8020-35.

157 Storhaug CL, Fosse SK, Fadnes LT. Country, regional, and global estimates for lactose malabsorption in adults: a systematic review and meta-analysis. Lancet Gastroenterol Hepatol 2017;2:738-46.

158 Zheng $X$, Chu H, Cong Y, et al. Self-reported lactose intolerance in clinic patients with functional gastrointestinal symptoms: prevalence, risk factors, and impact on food choices. Neurogastroenterol Motil 2015;27:1138-46.

159 Suarez FL, Savaiano DA, Levitt MD. A comparison of symptoms after the consumption of milk or lactose-hydrolyzed milk by people with self-reported severe lactose intolerance. N Engl J Med Overseas Ed 1995;333:1-4.

160 Böhmer CJM, Tuynman HARE. The effect of a lactose-restricted diet in patients with a positive lactose tolerance test, earlier diagnosed as irritable bowel syndrome: a 5-year follow-up study. Eur J Gastroenterol Hepatol 2001;13:941-4.

161 Corlew-Roath M, Di Palma JA. Clinical impact of identifying lactose maldigestion or fructose malabsorption in irritable bowel syndrome or other conditions. South Med $\mathrm{J}$ 2009;102:1010-2.

162 Parker TJ, Woolner JT, Prevost AT, et al. Irritable bowel syndrome: is the search for lactose intolerance justified? Eur J Gastroenterol Hepatol 2001;13:219-25.

163 Berg LK, Fagerli E, Martinussen M, et al. Effect of fructose-reduced diet in patients with irritable bowel syndrome, and its correlation to a standard fructose breath test. Scand J Gastroenterol 2013;48:936-43.

164 Halmos EP, Power VA, Shepherd SJ, et al. A diet low in fodmaps reduces symptoms of irritable bowel syndrome. Gastroenterology 2014;146:67-75.

165 Melchior C, Gourcerol G, Déchelotte P, et al. Symptomatic fructose malabsorption in irritable bowel syndrome: a prospective study. United Eur Gastroenterol J 2014;2:131-7.

166 Hauer-Jensen M, Denham JW, Andreyev HJN. Radiation enteropathy:pathogenesis, treatment and prevention. Nat Rev Gastroenterol Hepatol 2014;11:470-9.

167 Dinning PG, Szczesniak MM, Cook IJ. Twenty-four hour spatiotemporal mapping of colonic propagating sequences provides pathophysiological insight into constipation. Neurogastroenterol Motil 2008:20:1017-21.

168 Lindberg $\mathrm{G}$, Tornblom $\mathrm{H}$, Iwarzon $\mathrm{M}$, et al. Full-thickness biopsy findings in chronic intestinal pseudo-obstruction and enteric dysmotility. Gut 2009;58:1084-90.

169 Posserud I, Stotzer P-0, Bjornsson ES, et al. Small intestinal bacterial overgrowth in patients with irritable bowel syndrome. Gut 2007;56:802-8.

170 Soffer EE, Bruck R, Bar-Meir S. The role of short-term multilumen duodenojejunal manometry in patients with intestinal motor dysfunction. Gastroenterol Clin Biol 1988;12:123-5.

171 Stanghellini V, Cogliandro R, Cogliandro L, et al. Clinical use of manometry for the diagnosis of intestinal motor abnormalities. Dig Liver Dis 2000;32:532-41.

172 Marciani L, Cox EF, Hoad CL, et al. Postprandial changes in small bowel water content in healthy subjects and patients with irritable bowel syndrome. Gastroenterology 2010;138:469-77. 77 e1.

173 Fine KD, Fordtran JS. The effect of diarrhea on fecal fat excretion. Gastroenterology 1992;102:1936-9.

174 Thomas PD, Forbes A, Green J, et al. Guidelines for the investigation of chronic diarrhoea, 2nd edition. Gut 2003;52:1v-15.

175 Lankisch P, Dröge M, Hofses S, et al. Steatorrhoea: you cannot trust your eyes when it comes to diagnosis. Lancet 1996;347:1620-1.

176 Conwell DL, Lee LS, Yadav D, et al. American Pancreatic Association practice guidelines in chronic pancreatitis: evidence-based report on diagnostic guidelines. Pancreas 2014;43:1143-62.

177 Frulloni L, Falconi M, Gabbrielli A, et al. Italian consensus guidelines for chronic pancreatitis. Dig Liver Dis 2010;42(Suppl 6):S381-\$406.
178 Hoffmeister A, Mayerle J, Beglinger C, et al. English language version of the S3-consensus guidelines on chronic pancreatitis: definition, aetiology, diagnostic examinations, medical, endoscopic and surgical management of chronic pancreatitis. Z Gastroenterol 2015;53:1447-95.

179 Smith RC, Smith SF, Wilson J, et al. Summary and recommendations from the Australasian guidelines for the management of pancreatic exocrine insufficiency. Pancreatology 2016;16:164-80.

180 Lankisch PG, Schmidt I, Konig H, et al. Faecal elastase 1: Not helpful in diagnosing chronic pancreatitis associated with mild to moderate exocrine pancreatic insufficiency. Gut 1998;42:551-4.

181 Loser C, Mollgaard A, Folsch UR. Faecal elastase 1: a novel, highly sensitive, and specific tubeless pancreatic function test. Gut 1996;39:580-6.

182 Sonwalkar SA, Holbrook IB, Phillips I, et al. A prospective, comparative study of the para-aminobenzoic acid test and faecal elastase 1 in the assessment of exocrine pancreatic function. Aliment Pharmacol Ther 2003;17:467-71.

183 Elphick DA, Kapur K. Comparing the urinary pancreolauryl ratio and faecal elastase- 1 as indicators of pancreatic insufficiency in clinical practice. Pancreatology 2005;5(2-3):196-200.

184 Buscail L, Escourrou J, Moreau J, et al. Endoscopic ultrasonography in chronic pancreatitis: a comparative prospective study with conventional ultrasonography, computed tomography, and ERCP. Pancreas 1995;10:251-7.

185 Kusano S, Kaji T, Sugiura Y, et al. Ct demonstration of fibrous stroma in chronic pancreatitis: pathologic correlation. J Comput Assist Tomogr 1999;23:297-300.

186 Arasaradnam RP, Wicaksono A, O'Brien H, et al. Noninvasive diagnosis of pancreatic cancer through detection of volatile organic compounds in urine. Gastroenterology 2018;154:485-7.

187 Testoni PA, Mariani A, Curioni S, et al. Pancreatic ductal abnormalities documented by secretin-enhanced mrcp in asymptomatic subjects with chronic pancreatic hyperenzymemia. Am J Gastroenterol 2009;104:1780-6.

188 Testoni PA, Mariani A, Curioni S, et al. MRCP-secretin test-guided management of idiopathic recurrent pancreatitis: long-term outcomes. Gastrointest Endosc 2008;67:1028-34.

189 Bian Y, et al. Quantification of pancreatic exocrine function of chronic pancreatitis with secretin-enhanced MRCP. World J Gastroenterol 2013;19:7177-82.

190 Sanyal R, Stevens T, Novak E, et al. Secretin-enhanced MRCP: review of technique and application with proposal for quantification of exocrine function. AJR Am J Roentgenol 2012;198:124-32.

191 Gillams A, Pereira S, Webster G, et al. Correlation of MRCP quantification (MRCPQ) with conventional non-invasive pancreatic exocrine function tests. Abdom Imaging 2008;33:469-73

192 Manfredi R, Perandini S, Mantovani W, et al. Quantitative MRCP assessment of pancreatic exocrine reserve and its correlation with faecal elastase- 1 in patients with chronic pancreatitis. Radiol Med 2012;117:282-92.

193 Trikudanathan G, Walker SP, Munigala S, et al. Diagnostic performance of contrastenhanced MRI with secretin-stimulated MRCP for non-calcific chronic pancreatitis: a comparison with histopathology. Am J Gastroenterol 2015;110:1598-606.

194 Seicean A. Endoscopic ultrasound in chronic pancreatitis: Where are we now? World J Gastroenterol 2010;16:4253-63.

195 Stevens T, Conwell DL, Zuccaro G, et al. A prospective crossover study comparing secretin-stimulated endoscopic and Dreiling tube pancreatic function testing in patients evaluated for chronic pancreatitis. Gastrointest Endosc 2008;67:458-66.

196 Stevens T, Dumot JA, Parsi MA, et al. Combined endoscopic ultrasound and secretin endoscopic pancreatic function test in patients evaluated for chronic pancreatitis. Dig Dis Sci 2010;55:2681-7.

197 Mariani A, Arcidiacono PG, Curioni S, et al. Diagnostic yield of ERCP and secretinenhanced MRCP and EUS in patients with acute recurrent pancreatitis of unknown aetiology. Dig Liver Dis 2009;41:753-8.

198 Pungpapong S, Wallace MB, Woodward TA, et al. Accuracy of endoscopic ultrasonography and magnetic resonance cholangiopancreatography for the diagnosis of chronic pancreatitis. J Clin Gastroenterol 2007;41:88-93.

199 Go VL, Dimagno EP. Assessment of exocrine pancreatic function by duodenal intubation. Clin Gastroenterol 1984;13:701-15.

200 Lankisch PG. Function tests in the diagnosis of chronic pancreatitis. Critical evaluation. Int J Pancreatol 1993:14:9-20.

201 Axon AT, Classen M, Cotton PB, et al. Pancreatography in chronic pancreatitis: international definitions. Gut 1984:25:1107-12

202 Valentini M, Cavallini G, Vantini I, et al. A comparative evaluation of endoscopic retrograde cholangiopancreatography and the secretin-cholecystokinin test in the diagnosis of chronic pancreatitis: a multicentre study in 124 patients. Endoscopy 1981;13:64-7.

203 Niederau C, Grendell JH. Diagnosis of chronic pancreatitis. Gastroenterology 1985;88:1973-95.

204 Keller J, Meier V, Wolfram KU, et al. Sensitivity and specificity of an abbreviated (13) C-mixed triglyceride breath test for measurement of pancreatic exocrine function. United Eur Gastroenterol I 2014;2:288-94.

205 Choung RS, Ruff KC, Malhotra A, et al. Clinical predictors of small intestinal bacterial overgrowth by duodenal aspirate culture. Aliment Pharmacol Ther 2011;33:1059-67. 
206 Browning GG, Buchan KA, Mackay C. The effect of vagotomy and drainage on the small bowel flora. Gut 1974;15:139-42.

207 Brägelmann R, Armbrecht U, Rosemeyer D, et al. Small bowel bacterial overgrowth in patients after total gastrectomy. Eur J Clin Invest 1997;27:409-16.

208 Lewis SJ, Potts LF, Malhotra R, et al. Small bowel bacterial overgrowth in subjects living in residential care homes. Age Ageing 1999;28:181-5.

209 Riordan SM, Mclver CJ, Wakefield D, et al. Small intestinal bacterial overgrowth in the symptomatic elderly. Am J Gastroenterol 1997:92:47-51.

210 Saltzman JR, Kowdley KV, Pedrosa MC, et al. Bacterial overgrowth without clinical malabsorption in elderly hypochlorhydric subjects. Gastroenterology 1994;106:615-23.

211 Corazza GR, Menozzi MG, Strocchi A, et al. The diagnosis of small bowel bacterial overgrowth. Reliability of jejunal culture and inadequacy of breath hydrogen testing. Gastroenterology 1990;98:302-9.

212 Rhodes JM, Middleton P, Jewell DP. The lactulose hydrogen breath test as a diagnostic test for small-bowel bacterial overgrowth. Scand I Gastroenterol 1979;14:333-6.

213 King CE, Toskes PP. Breath tests in the diagnosis of small intestine bacterial overgrowth. Crit Rev Clin Lab Sci 1984:21:269-81.

214 Zhao J, Zheng X, Chu H, et al. A study of the methodological and clinical validity of the combined lactulose hydrogen breath test with scintigraphic oro-cecal transit test for diagnosing small intestinal bacterial overgrowth in IBS patients. Neurogastroenterol Motil 2014;26:794-802

215 Thompson DG, O'Brien JD, Hardie JM. Influence of the oropharyngeal microflora on the measurement of exhaled breath hydrogen. Gastroenterology 1986:91:853-60.

216 Saltzberg DM, Levine GM, Lubar C. Impact of age, sex, race, and functional complaints on hydrogen (H2) production. Dig Dis Sci 1988;33:308-13.

217 Erdogan A, Rao SS, Gulley D, et al. Small intestinal bacterial overgrowth: duodenal aspiration vs glucose breath test. Neurogastroenterol Motil 2015;27:481-9.

218 Riordan SM, Mclver CJ, Walker BM, et al. The lactulose breath hydrogen test and small intestinal bacterial overgrowth. Am J Gastroenterol 1996;91:1795-803.

219 Lin EC, Massey BT. Scintigraphy demonstrates high rate of false-positive results from glucose breath tests for small bowel bacterial overgrowth. Clin Gastroenterol Hepatol 2016;14:203-8.

220 Yu D, Cheeseman F, Vanner S. Combined oro-caecal scintigraphy and lactulose hydrogen breath testing demonstrate that breath testing detects oro-caecal transit, not small intestinal bacterial overgrowth in patients with IBS. Gut 2011;60:334-40.

221 Stotzer PO, Brandberg A, Kilander AF. Diagnosis of small intestinal bacterial overgrowth in clinical praxis: a comparison of the culture of small bowel aspirate, duodenal biopsies and gastric aspirate. Hepatogastroenterology 1998;45:1018-22.
222 Attar A, Flourié B, Rambaud JC, et al. Antibiotic efficacy in small intestinal bacterial overgrowth-related chronic diarrhea: a crossover, randomized trial. Gastroenterology 1999;117:794-7.

223 Sanders DS, Hurlstone DP, Brown S. Does coeliac disease affect colorectal practice? Int J Colorectal Dis 2007;22:565-70.

224 Cowlam S, Vinayagam R, Khan U, et al. Blinded comparison of faecal loading on plain radiography versus radio-opaque marker transit studies in the assessment of constipation. Clin Radiol 2008:63:1326-31.

225 Armbrecht U, Lundell L, Lindstedt $\mathrm{G}$, et al. Causes of malabsorption after total gastrectomy with Roux-en-Y reconstruction. Acta Chir Scand 1988;154:37-41.

226 Swan RW. Stagnant loop syndrome resulting from small-bowel irradiation injury and intestinal by-pass. Gynecol Oncol 1974;2:441-5.

227 Castiglione F, Del Vecchio Blanco G, Rispo A, et al. Orocecal transit time and bacteria overgrowth in patients with Crohn's disease. J Clin Gastroenterol 2000;31:63-6.

228 Costi R, Randone B, Violi V, et al. Cholecystocolonic fistula: facts and myths. A review of the 231 published cases. J Hepatobiliary Pancreat Surg 2009;16:8-18.

229 Dukowicz AC, Lacy BE, Levine GM. Small intestinal bacterial overgrowth: a comprehensive review. Gastroenterol Hepatol 2007;3:112-22.

230 Eriksson B, Oberg K, Skogseid B. Neuroendocrine pancreatic tumors. Clinical findings in a prospective study of 84 patients. Acta Oncol 1989;28:373-7.

231 Reid MD, Balci S, Saka B, et al. Neuroendocrine tumors of the pancreas: current concepts and controversies. Endocr Pathol 2014;25:65-79.

232 Mekhjian HS, O'Dorisio TM. VIPoma syndrome. Semin Oncol 1987;14:282-91.

233 Ganguli PC, Cullen DR, Irvine WJ. Radioimmunoassay of plasmagastrin in pernicious anaemia, achlorhydria without pernicious anaemia, hypochlorhydria, and in controls. Lancet 1971:1:155-8.

234 Caplin ME, Buscombe JR, Hilson AJ, et al. Carcinoid tumour. Lancet 1998;352:799-805.

235 Ramage JK, Ahmed A, Ardill J, et al. Guidelines for the management of gastroenteropancreatic neuroendocrine (including carcinoid) tumours (NETs). Gut 2012;61:6-32.

236 Eriksson B, Arnberg H, Lindgren PG, et al. Neuroendocrine pancreatic tumours: clinical presentation, biochemical and histopathological findings in 84 patients. Intern Med 1990:228:103-13.

237 Halperin DM, Shen C, Dasari A, et al. Frequency of carcinoid syndrome at neuroendocrine tumour diagnosis: a population-based study. Lancet Oncol 2017; 18:525-34

238 Afzalpurkar RG, Schiller LR, Little KH, et al. The self-limited nature of chronic idiopathic diarrhea. N Engl J Med 1992;327:1849-52.

239 Hungin AP, Paxman L, Koenig K, et al. Prevalence, symptom patterns and management of episodic diarrhoea in the community: a population-based survey in 11 countries. Aliment Pharmacol Ther 2016:43:586-95. 